\title{
„Wissenschaft und Kunst sind, wie selten, eine glückliche Verbindung eingegangen“. - Das Rom-Panorama von Josef Bühlmann im Kontext des 19. Jahrhunderts ${ }^{1}$
}

\author{
VALENTIN KOCKEL
}

Den Anlass zu dem Leipziger Kolloquium bot das von Yadegar Asisi wieder belebte, überarbeitete und auch verfremdete Rom-Panorama, das ab 1886 von dem Architekten Josef Bühlmann und dem Historienmaler Alexander von Wagner angefertigt wurde (Faltblatt 1 und 2). Es zeigt die antike Stadt im Jahre 312, am Tag nach dem Sieg Konstantins über Maxentius an der Milvischen Brücke. Konstantin eilt mit seinem Gefolge im Triumphzug vom Forum auf das Capitol, wo ihn die Priester des römischen Staatsgottes erwarten. Mehrere Nebenhandlungen begleiten das Geschehen: Eine Gruppe von Christen zeigt die Standarte, das sogenannte Labarum; Soldaten stürzen eine Statue von Konstantins Gegner; Priester vollziehen ein heidnisches Opferritual vor dem Tempel der Iuno Moneta. Als riesiges Historienbild stellt das Panorama damit den - historisch nicht überlieferten - Augenblick dar, in dem sich der Wechsel von der heidnischen zur christlichen Antike abzeichnet. In dem folgenden Beitrag soll jedoch vor allem der Anteil des Architekten Bühlmann an diesem Werk behandelt und innerhalb der Panoramamalerei des 19. Jahrhunderts verortet werden. Sein ,historischer' Rundblick erweist sich als wichtiges Beispiel früher bauhistorischer Forschung und Visualisierung. Bühlmanns Entwurf der Stadt Rom wird außerdem in eine Reihe mit zeichnerischen Rekonstruktionen antiker Stadtbilder gestellt, deren Erforschung damals noch am Anfang stand.

Als Robert Barker am 17. Juni 1787 das Patent für eine Ausstellungstechnik erhielt, die er auf französisch „La nature à coup d'oeuil“ nannte, bereitete er damit den Weg für eine Entwicklung, in der mehr denn je die möglichst überwältigende Wirklichkeitsnähe zu einem Thema bildnerischen Schaffens avancierte. Schon bald wurde der kom-

\footnotetext{
${ }^{1}$ Für Hinweise und Hilfe danke ich Klaus Anton Altenbuchner, Renate Miller-Gruber, Wolf Koenigs, Winfried Nerdinger, Anja Schmidt, Denis Stante, Hilde Strobl, Ulrike Wulf-Rheidt.

Nachtrag: Vom Verf. erschien mittlerweile ein Beitrag, in dem die Panoramen ebenfalls eine Rolle spielen. V. Kockel, Rekonstruktion und Rezeption - Die Rekonstruktion antiker Stadtbilder und ihre Verbreitung, in: W. Nerdinger (Hrsg.), Geschichte der Rekonstruktion - Konstruktion der Geschichte. Ausstellungskatalog München (München 2010) 96-113.381-403.
} 
plizierte französische Begriff durch den pseudogriechischen Neologismus ,Panorama ersetzt, ein Wort, das in fast allen Sprachen gut zu artikulieren ist und sicher auch deshalb zum Erfolg der Erfindung beitrug. Über den Siegeszug des neuen Mediums in den Großstädten Europas und Amerikas sind in den letzten dreißig Jahren einige ausführliche Darstellungen erschienen. ${ }^{2}$ In Deutschland führte vor allem die 1993 in

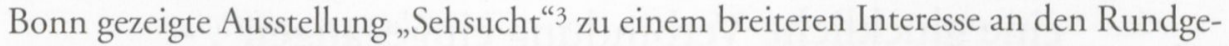
mälden und seither wurden wieder einige der historischen Panoramen restauriert und der Öffentlichkeit zugänglich gemacht. ${ }^{4}$ In Bonn hatte Yadegar Asisi auch eine erstenoch sehr kleine - überarbeitete Fassung von Bühlmanns Panorama aus dem Jahr 1888 ausgestellt. Aus diesem Versuch entstand letztlich das Leipziger Rom-Panorama. ${ }^{5}$ Bevor wir uns ihm zuwenden, sollen aber noch einige Grundsätze reflektiert werden, die den spezifischen Charakter von Panoramen ausmachen und bei der Einschätzung der Arbeit Bühlmanns berücksichtigt werden müssen.

Es ist stets zu Recht betont worden, dass Barkers Leistung, und letztlich auch die Berechtigung für die Patentierung seiner Idee, nicht so sehr in der Form des Rundgemäldes als solchem bestand - Vergleichbares gab es bereits vorher -, sondern vielmehr in der Art seiner Präsentation. Der Betrachter sollte durch die Ausstellungstechnik möglichst ohne Ablenkung in das dargestellte Thema einbezogen werden, wofür man durch eine immer raffiniertere Abdunkelung und Lichtführung sorgte. ${ }^{6}$ Der Betrachter wurde damit zum Bezugspunkt des Panoramas. Wie im vorkopernikanischen Kosmos steht er im Mittelpunkt einer Welt, die auf die gekrümmte innere Schale des Ausstellungsbaus projiziert wird. Praktisch geht es dabei auch um komplizierte Verzerrungen in der Darstellungsweise, deren Konstruktion erst geheim gehalten, dann aber im Rahmen der Ausbildung zur Perspektivdarstellung auch an den Akademien gelehrt

${ }^{2}$ S. Oettermann, Das Panorama. Die Geschichte eines Massenmediums (Frankfurt am Main 1980; engl. 1997) - immer noch unverzichtbar mit der materialreichsten Sammlung; S. Bordini, Storia del panorama. La visione totale nella pittura del XIX secolo (Rom 1984); M.-L. von Plessen (Hrsg.), Sehsucht. Das Panorama als Massenunterhaltung des 19. Jahrhunderts. Ausstellungskatalog Bonn (Basel 1993); B. Comment, Das Panorama. Die Geschichte einer vergessenen Kunst (Berlin 2000; frz. 1993; engl. 1999).

3 Sehsucht (Anm. 2).

${ }_{4}$ Zum Beispiel das restaurierte Jerusalem-Panorama in Altötting, die neu entwickelten Panoramen von Yadegar Asisi in Leipzig (Everest) und Dresden (Dresden 1756), sowie Johann Michael Sattlers Salzburg-Panorama aus dem Jahr 1829, das seit 2006 wieder in Salzburg zu sehen ist. Eine Liste zugänglicher Panoramen findet sich auch in dem im Auftrag des International Panorama Council herausgegebenen Bildband: T. Rombout (Hrsg.), The Panorama Phenomenon (Den Haag 2006).

5 Sehsucht (Anm. 2) 172f. Nr. II.102f. Eine transparente, kleine' Version war auch 2007 in der Konstantin-Ausstellung in Trier zu sehen. Ein 1996 für die Grünanlagen der Via dei Fori Imperiali in Rom konzipierter Schauraum wurde nicht realisiert. Vgl. Geo-Special Rom, Oktober 1996, 60-65.

${ }^{6}$ Dabei ergaben sich auch statische Probleme: So entwickelte Jakob Ignaz Hittorf 1831 eigens eine neuartige Dachkonstruktion, um eine Ausstellungs-Rotunde an den Champs Elysées mit einer Spannweite von 38 m stützenfrei überdachen zu können. Comment (Anm. 2) 47-48 Abb. 19-20. 
wurde. ${ }^{7}$ Der Besucher stand idealerweise nicht vor, sondern im Bild. ${ }^{8}$ So verstanden ist das Panorama also im eigentlichen Sinn keine Bildgattung, sondern eine Technik, eine Hülle, besser noch ein Medium, mit dem die traditionellen Genera wie Landschaftsmalerei, Historienbild oder, spezifischer, Schlachtenbild in neuer Weise für ein breites Publikum aufbereitet werden - deshalb auch das von Stephan Oettermann glücklich eingeführte Schlagwort vom Panorama als dem ersten ,Massenmedium'.

Die Intention, den Betrachter möglichst nahtlos in die virtuelle Welt der Bilder einzubinden, und die dafür entwickelte, Technik' hatten formale und inhaltliche Konsequenzen für die Gestaltung der Bilder und deren Aussage. Für die Form der Bilder ist zunächst die Wahl des Standortes und die Enge des Bezugs zum dargestellten Objekt wesentlich. Der Betrachter steht meist leicht erhöht, zum Beispiel auf einem Turm oder einem (Feldherren-) Hügel, um einen besseren Überblick zu gewinnen. Rückt sein Standort noch selbst ins Bild, ergeben sich starke Verzerrungen, die oft ganz bewusst betont und als kompositorische Effekte eingesetzt werden. ${ }^{9}$ Die erklärenden Broschüren wählen deshalb vor allem im beginnenden 19. Jahrhundert nicht selten stark anamorphische Darstellungsformen, die den kaum durchschaubaren, verblüffenden Charakter des Bildes noch unterstreichen und das Virtuosentum des Malers betonen. ${ }^{10}$ Hält der Betrachter jedoch Abstand und wird sein Blick eher horizontal gerichtet, dann entwickeln sich weit gezogene Landschaften, in denen der einzelne Gegenstand schrumpft und der Himmel schon von der Malfläche her eine große Bedeutung gewinnt - wir würden heute von Weitwinkeleffekten sprechen.

Die Einbindung des Betrachters führt aber auch zu einem inhaltlichen Postulat, das die ganze Tradition der Panoramamalerei durchzieht. Ich möchte es als ein immanentes Versprechen von absoluter Authentizität bezeichnen. Nicht umsonst beginnen die meisten Panorama-Schausteller zuerst mit Abbildungen jener Stadt, in der das Werk selbst gezeigt wird. Was zunächst widersinnig erscheinen mag, bezog seinen Reiz gerade aus der Überprüfbarkeit der Fakten für jedermann. Der Betrachter ist gleichzeitig der beste Kenner des Dargestellten und noch das einfachste Dienstmädchen kann theoretisch die Dachgaube seiner Schlafkammer identifizieren. Dieser Anspruch

${ }^{7}$ Der deutsche ,Erfinder' des Panoramas, Johann Adam Breysig (1766-1831), lehrte als akademischer Zeichenlehrer in Magdeburg und Danzig und bildete dort unter anderem Karl Georg Enslen (s.u.) aus. Oettermann (Anm. 2) 150-156.

${ }^{8}$ An dieser Stelle soll nur kurz darauf verwiesen werden, dass die heute so beliebte und insbesondere durch digitale Techniken vereinfachte Panoramafotografie gerade diesen Anspruch nicht erfüllt. Sie zeigt zwar idealerweise den Rundumblick, projiziert ihn aber in die Fläche und hält den Betrachter damit in Distanz. Vgl. z.B. N. Meers, Faszination Panoramafotografie (München 2003) 23-24 zur Entwicklung fotografischer ,Panoramen'.

9 Vgl. das Dach der Albion-Mills, das als Standort von Robert Barkers London-Panorama 1792 gewählt wurde (Comment [Anm. 2] 22-23 Abb. 7) oder auch das Dach der Werderschen Kirche im Vordergrund von Eduard Gärtners Berlin-Panorama von 1834; Oettermann (Anm. 2) 171-172; Sehsucht (Anm. 2) 224-225 Nr. IV.29.

${ }^{10} \mathrm{Vgl}$. Comment (Anm. 2) 164-165 Abb. 83. 85 (Paris 1804 und London 1845); Bordini (Anm. 2) bildet besonders viele dieser Begleithefte ab. 




Abb. 1 Luigi Rossini, Drei Blätter des „Panorama di Roma antica e moderna“. Aus: I Sette Colli di Roma antica e moderna (Rom 1827-1829) Taf. 27-29

auf, wie wir heute sagen würden, fotografische Genauigkeit galt später sinngemäß auch für andere Darstellungen. Vor allem bei den Schlachtenbildern wurden in den Begleittexten gern möglichst hochrangige Augenzeugen des Geschehens als Berater zitiert, die durch ihre Person die Authentizität der Abbildung bezeugten. Wurden, wie vermehrt im späten 19. Jahrhundert, zum Teil weit zurückliegende historische Ereignisse dargestellt, dann galt wie beim Historienbild allgemein, die Regel des vraisemblable, des Wahrscheinlichen für Kostüm und Umgebung. Je mehr der Maler diesem Anspruch genügte, je umfassender seine Vorstudien den Ort selbst und die literarische Überlieferung einbezogen, desto größer war das Lob für seine Arbeit. Dieser Aspekt wird auch für die Wertschätzung von Bühlmanns Panorama wichtig sein.

$\mathrm{Zu}$ den beliebtesten Themen der Panoramabilder des frühen 19. Jahrhunderts gehören neben aktuellen historischen Ereignissen vor allem Ansichten von Städten und spektakulärer Landschaften überall auf der Welt. Alexander von Humboldt empfahl sogar in seinem „Kosmos“ die Panoramen als didaktisches Anschauungsmaterial. ${ }^{11}$ Natürlich gehörte zu den dargestellten Städten auch Rom, wobei die Ruinen nicht unbedingt im Mittelpunkt stehen mussten. So zeigte Louis Le Masson 1779 die Stadt in einem Rundgemälde von S. Pietro in Montorio aus - die antiken Ruinen liegen kaum sichtbar im Hintergrund. ${ }^{12}$ Das in London aufbewahrte Panorama Ludovico

11 Oettermann (Anm. 2) 33.

12 Comment (Anm. 2) 144 Nr. II.50 und Falttafeln S. 96-98. Bei dem Bild Le Massons handelt es sich sozusagen um ein Panorama, avant la lettre'. 


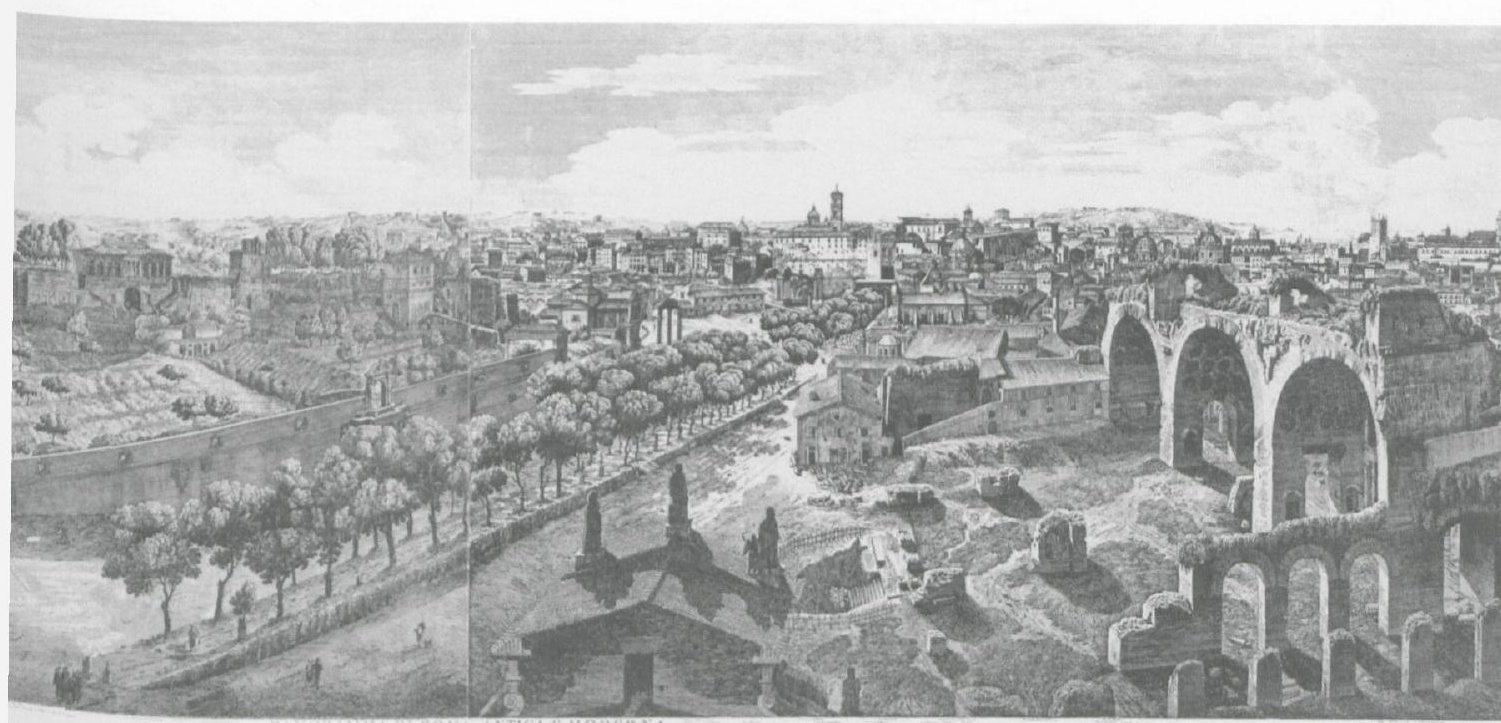

Caracciolos von 1824 hat dagegen eindeutig die antiken Denkmäler zum Thema, die aber wie in einem Bild Hubert Roberts zu einer neuen Wirklichkeit zusammen komponiert sind, um möglichst vorteilhaft zu erscheinen. ${ }^{13}$ Gleich das erste, im Jahr 1800 in Deutschland nach Zeichnungen von Johann Adam Breysig angefertigte Panorama zeigte Rom vom Palatin aus. ${ }^{14}$ Henry Aston Barker (1817) und Carl Georg Enslen (1820er Jahre) porträtierten Rom vom Turm des Senatorenpalastes aus, so dass die antike und die päpstliche Stadt einander gegenüber standen. ${ }^{15}$ Als eine Folge von vier Blättern fügte Luigi Rossini etwa gleichzeitig ein Panorama in sein Stichwerk „I Sette Colli di Roma antica e moderna" ein, das er von der Kirche S. Maria Nuova nahe dem Titusbogen aufgenommen hatte (Abb. 1). ${ }^{16}$ Ein weiteres, heute weitgehend ver-

${ }^{13}$ Das (Miniatur-) Panorama befindet sich im Victoria \& Albert Museum. Comment (Anm. 2) 196-200 Abb. 107-113.

14 Oettermann (Anm. 2) 153-156 mit Abb. 112.

15 Bordini (Anm. 2) 243 Abb. 105 (Barker); V. Kockel, Pompeji 360 . Die beiden Panoramen Carl Georg Enslens aus dem Jahr 1826 (Mailand 2006) 24 (Enslen, heute verloren).

${ }^{16}$ L. Rossini, I Sette Colli di Roma antica e moderna (Rom 1827-29) Taf. 27-30. Abbildung in: N. Pirazzoli, Luigi Rossini 1790-1856. Roma antica restaurata (Ravenna 1990) 190-191. Der von Rossini im Vorwort angekündigte „restauro dei medesimi (colli) secondo gli avanzi ..." beschränkt sich vornehmlich auf die Pläne der sieben Hügel. Ähnlich hieß es wohl auch schon im Aufruf zur Subskription. So steht im Kunstblatt 93 vom 20. 11. 1826, S. 372, dass in dem Werk unter anderem „ein Panorama von Rom, ebenfalls mit Restauration ..." zu erwarten sei. Rekonstruktionen finden sich nur vom Forum und ausschnitthaft vom Palatin. Auch die im Begleittext 




Abb. 2 Carl Sprosse, Titelblatt von Dr. Emil Braun's Panorama von Rom. (Leipzig 1851). Titelvignette

gessenes Panorama erschien 1851 als Mappenwerk auf Initiative des deutschen Archäologen Emil Braun. ${ }^{17}$ Doch anders als man bei einem Archäologen erwarten könnte, zeigt das in seinem Auftrag von Carl Sprosse von der Villa Ludovisi aus aufgenommene Panorama ein Rom, wie es grüner und malerischer nicht sein kann. ${ }^{18}$

zu den Tafeln 27-30 für künftige Zeiten angekündigten „altre due tavole aggiunte, ove saranno collocati altri restauri di grandiosi Edifici di Roma" scheinen nicht vollendet worden zu sein. Vorzeichnungen zu einem erweiterten Panorama von Forum und Capitol werden jedoch von Pirazzoli (diese Anm.) 93 Abb. 105 abgebildet. - Auf dieses Panorama machte mich Renate Miller-Gruber aufmerksam, für wichtige Auskünfte danke ich Anja Schmidt.

17 Dr. Emil Braun's Panorama von Rom. Aufgenommen vom Casino der Villa Ludovisi und in Kupfer gestochen von Carl Sprosse, Leipzig 1851. - Eingesehen wurde das Exemplar der Universitätsbibliothek Göttingen. Für Hilfe danke ich Daniel Graepler. - Braun leitete damals das Instituto di Corrispondenza Archeologica, den Vorläufer des Deutschen Archäologischen Instituts in Rom und war technischen Neuerungen wie zum Beispiel der Fotografie gegenüber ausgesprochen aufgeschlossen. Zu Braun (1809-1856) jetzt H. Schmidt - P. G. Schmidt, Emil Braun „ein Mann edelster Begabung von Herz und Geist“ (Altenburg 2010).

18 Carl Ferdinand Sprosse (1819-1914) war Architekturmaler, Zeichner und Radierer. In Rom hielt er sich von 1844 bis 1850 auf. Außer den neun Blättern des Rom-Panoramas sind zwölf Ansichten des Forum Romanum bekannt, ferner 32 Blätter mit Ansichten aus Rom und Umge- 
EXPLANATION of a VIEW of the RUINS of POMPEI
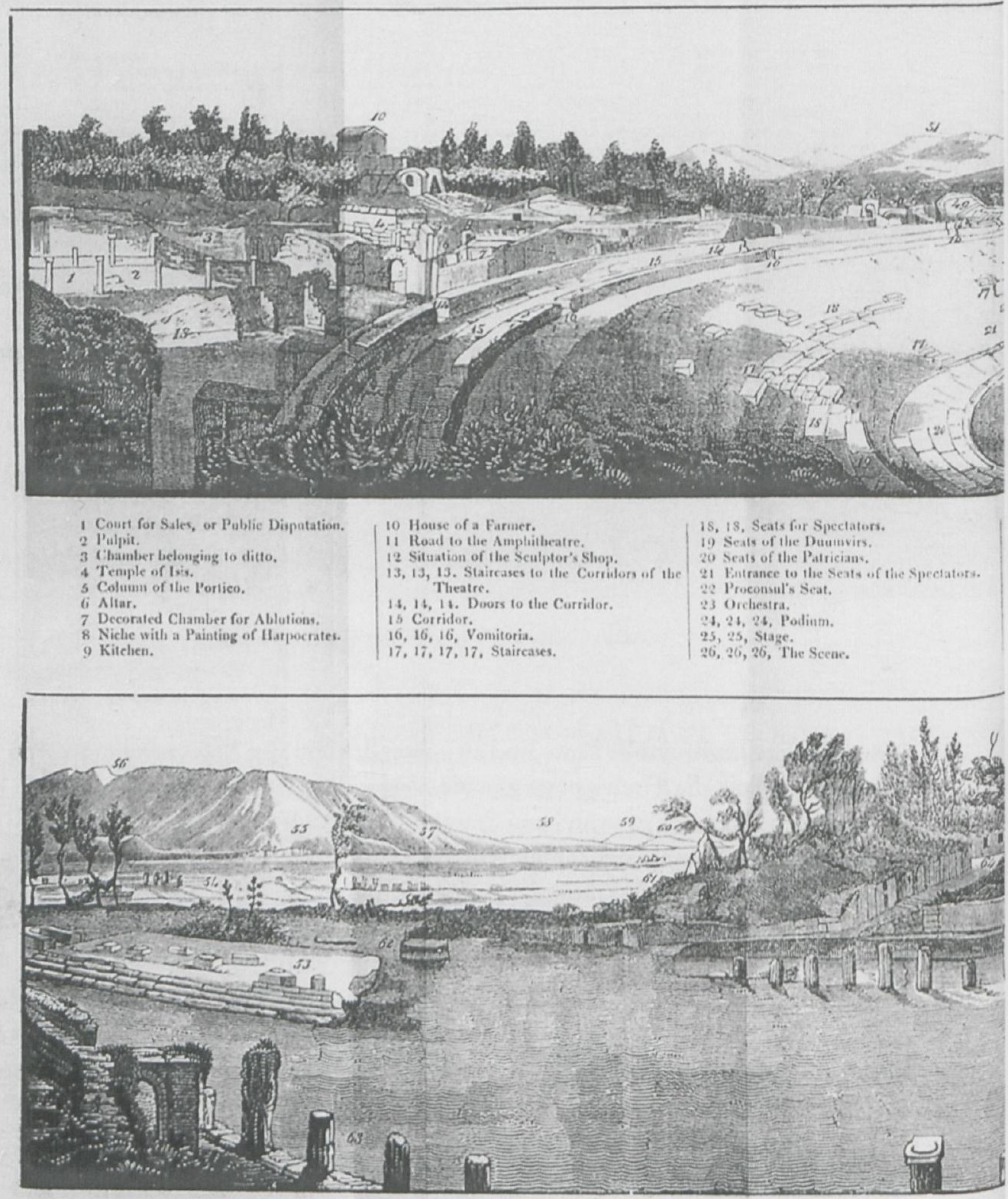

32 Sacred Pea for Caltur. 53 Temple of Hereules.

36 Site of Stabra.

ss Castell" a Mare.

56 Maunt lactarius.
37 Vico.

38 Sorrento.

\$9 Caje Minerva.

60 Island of Capri.

6i Rock of IIercules.
62 Circular Seat, commanding a beautiful and extensive View.

63 Portico of the Peribolus, or Court of the 'Teisple of Hercules.

64 Stret leating to Q. Caroline's Excavation.

S. Ainc alas m

Abb. 3 Thomas L. Donaldson, Explanation of a view of the city of Pompeii, exhibiting in the Panorama. Leicester-Square (London 1824) 
I, exhibiting at the PANORAMA, LEICESTER-SQUARE.



27 Royal Door.

28 Doors of the Strangers.

30 Postscenium Couniry.

31 Sestscenium.

Si Seats for the Women.

35 Eitrance to the Seats for the Women.

3. Blevated the Awning. placed mover on which were generally
peats.
35 Entrance to ilitto.

36 Enclesare attached to the Tenple of Hercules.

37 Alrars.

38 Circular Colonnale.

99 Greguano.

40 Lettere.

41 Mente S. Angelo.

4\% Guard's Quarters,
43 Forum Nundinarium, or Market.

4s Steps leading up to the Temple of II r rules

45 Back Wall to the Shops of ihe Fitum.

46 Postscenium of the euvered Ilicatre.

47,47 , Eutrances to ditte.

18, 48, Entrance to the Stage of ditto.

49 Spectator's Seats.

so Passages leadiag to ll:e coreted Theatre.

s1 The $A$ ppenimes.

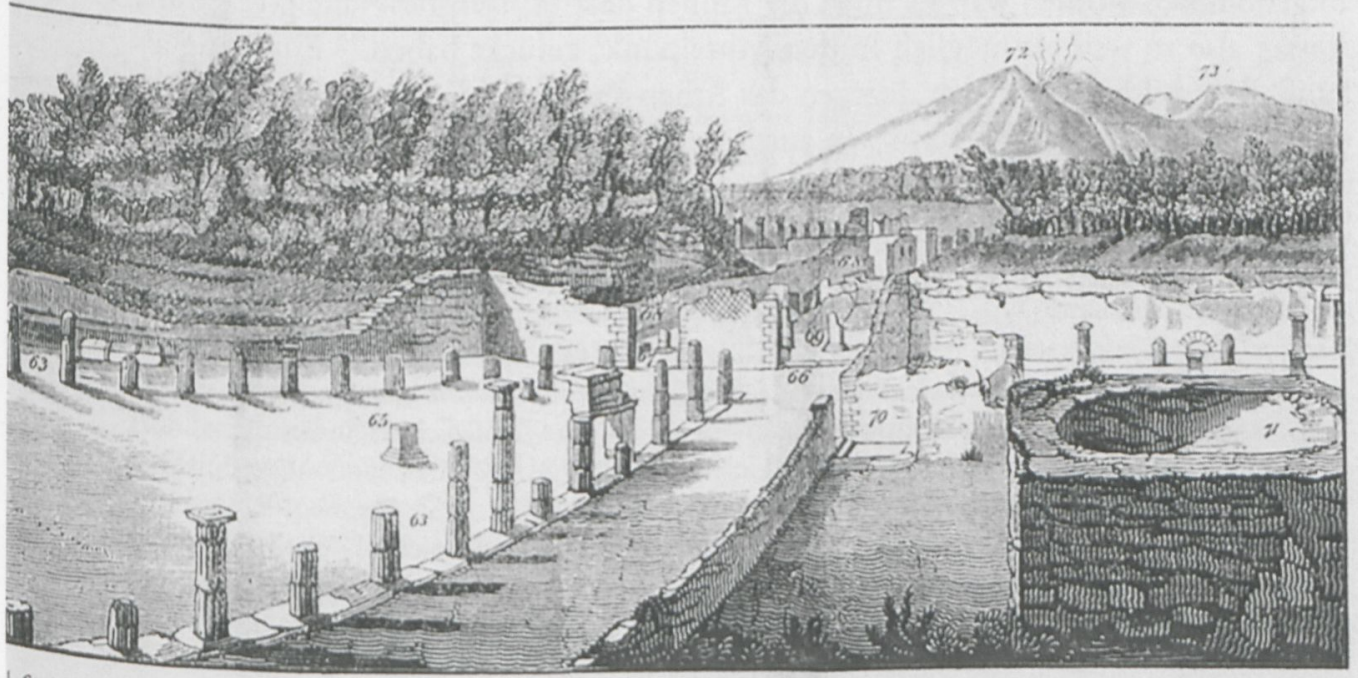

65 Pedestal for a Statue.

66 The Propylea Statue. 68 Strect leading to the ateater Forum,

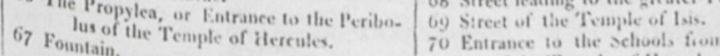

71 Reservoir.

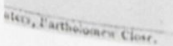

of tie Teuple of Hereul?

72 Crater of Vesuvius.




sent konnte angeblich den Stein entdecken, auf dem er sich von den Strapazen seiner Grand Tour erholt hatte. Von diesen Panoramen sind nur die Umzeichnungen für das obligatorische Begleitheft erhalten (Abb. 3). Besser dokumentiert ist dagegen ein Zimmer-Panorama des Malers Carl Georg Enslen (1792-1866). ${ }^{21}$ Es entstand 1826 und die teilweise kolorierten vorbereitenden Aquarelle werden heute in Lübeck aufbewahrt (Taf. 8a). Enslen hatte als Standort die Ecke der Theatercavea gewählt und betont mit der stark verkürzten Darstellung dieser Mauer den direkten Bezug des Betrachters zum Ort. Der unfertige Zustand der Blätter, auf denen die skizzenhaft angelegten Ruinen hinter der farbigen Landschaft zurücktreten, charakterisiert dieses Panorama deutlich als Landschaftsbild. Himmel und umgebende Landschaft nehmen oft mehr als die Hälfte der Bildhöhe ein, die Ruinen selbst erscheinen klein und unbedeutend. Pompeji ist damit ein malerischer Ort am gerühmten Golf von Neapel, dessen Ruinen den Reisenden zwar anziehen, aber - ebenso wie die liebevoll dargestellten Staffagefiguren neapolitanischer Landleute - vor allem den pittoresken Charakter dieser Region unterstreichen. Natürlich geht es dem Künstler um eine möglichst authentische Abbildung des Ist-Zustandes, eine Rekonstruktion der Ruinenstätte entsprach nicht seinen Intentionen und hätte auch seine Möglichkeiten als Maler völlig überfordert.

Seltener als Rom wurde Athen in einem Panorama porträtiert. In Paris sah man 1821 ein Rundgemälde des damals hochgeschätzten Spezialisten Pierre Prévost, das zwischen 1816 und 1819 von dem mittelalterlichen, Frankenturm' auf der Akropolis aufgenommen worden war. Es muss die Ruinen dort in nächster Nähe gezeigt, die Antike also so weit als möglich in den Mittelpunkt gerückt haben. ${ }^{22}$ Eindeutig als Landschaftsbild versteht sich dagegen das Athen-Panorama Ferdinand Stademanns von 1835 (Abb. 4). Stademann, ein aus Bayern stammender Beamter König Ottos und zeichnerisch begabt, ließ sich auf dem Felsen des Nymphenheiligtums nieder und zeichnete ein Athen in karger Umgebung, das uns bei dem Gedanken an den heutigen Großstadt-Moloch besonders anrührt. ${ }^{23}$ Zwischen den weiten und kahlen

${ }^{21}$ Kockel (Anm. 15). Eine Kuriosität stellt ein aus z. T. übermalten Photos zusammengesetztes ,Panorama' dar, das einzelne Ansichten der Stadt mit jeweils unterschiedlichem Aufnahmestandort zu einem fiktiven Ganzen zusammenfügt. Holger Petersen, Pompeji und seine Ausgrabungen. Rundgemälde (München 1891) und spätere Auflagen. Abgebildet in M. Pagano - R. Prisciandaro, Studio sulle provenienze degli oggetti rinvenuti negli scavi borbonici del Regno di Napoli I (Castellammare di Stabia 2006) Abb. 15a-i nach S. 232. Bibliographischer Nachweis auch in: Nuova Bibliografia Pompeiana II (Rom 1998) 923 Nr. 10565.

${ }^{22}$ Lob und Kritik dazu in Schorns Kunstblatt 2, 1821, 341-343 (nach Oettermann [Anm. 2] 122); Comment (Anm. 2) 46. Pierre Prévost (1764-1823) galt als hervorragender Kolorist. Sein Panorama-Gebäude am Montmartre wurde bald nach seinem Tod geschlossen.

${ }^{23}$ F. Stademann, Panorama von Athen (München 1841). Ein leicht verkleinerter Nachdruck mit einem Vorwort von Frank Brommer erschien 1977 in Mainz. - Stademann (1791-1872) gehörte zum ersten bayerischen Kontingent in Griechenland. Der Archäologe Friedrich von Thiersch äußerte sich sehr positiv über die Genauigkeit des Panoramas, an Hand dessen man wissenschaftliche Untersuchungen knüpfen könne (Vorwort). Zu Stademanns Panorama zuletzt 
Hügeln liegt die kleine Provinzstadt, selbst die Akropolis verschwindet in der dominanten Landschaft. Dieses Panorama war von Beginn an dafür konzipiert, als Lithografie in einer Mappe zu erscheinen. Neben Lob für seine Genauigkeit erntete Stademann aber mit gleicher Begründung auch Kritik. Er habe, so bemerkte der Leiter des Antikendienstes Ludwig Ross, mit ,ängstlichem Fleiße“ sein großes Panorama von Athen gezeichnet. So transportierte man in den Augen von Ross also nicht die Größe der Antike! ${ }^{24}$

Auch Ägypten geriet in den Blick der Panorama-Künstler. So stellte zum Beispiel Robert Burford 1835 eine Ansicht der Ruinen von Theben aus. ${ }^{25}$ Einen Rundblick von einer der Pyramiden in Gizeh zeigt ein Panorama, das der großen wissenschaftlichen Publikation des deutschen Ägyptologen Richard Lepsius beigegeben wurde (Taf. 8b). 1842 bis 1846 war Lepsius in Ägypten gereist. Dabei entstanden mehrere Rundansichten, die im topografischen Teil der bis 1856 erscheinenden Tafelbände seiner „Denkmäler aus Aegypten und Äthiopien“ lagen. Auf vier einzelne Farblithografien verteilt, bilden sie dennoch insgesamt ein Ganzes. ${ }^{26}$ Ähnliche Panoramen, nun aber meist nach Fotos gezeichnet, blieben bis in das 20. Jahrhundert für wissenschaftliche Publikationen gebräuchlich. Auf einzelne Blätter verteilt, wird in großen Mappenwerken eine Rundumansicht angeboten, die sich nur im Kopf des Betrachters zusammenfügen kann und deren Autoren gar nicht anstreben, eine räumliche Illusion zu generieren. Die panoramatische Abbildung - von einem wirklichen Panorama kann man nicht sprechen - gewann damit für einige Zeit Bedeutung als umfassende Dokumentationsform. ${ }^{27}$

H. Schulten, in: R. Baumstark (Hrsg.), Das neue Hellas, Griechen und Bayern zur Zeit Ludwigs I. Ausstellungskatalog München (München 1999) 473 Nr. 328; 569-573 Nr. 428 (Abbildungen aller Blätter). Dort auch die kritische Äußerung von Ludwig Ross.

${ }^{24}$ Ebenfalls als Mappenwerk entstand 1833 ein Athen-Panorama der englischen Zeichnerin Selina Bracebridge (1800-1874), das 1836 als „Panoramic scetch of Athens" publiziert wurde (nicht gesehen). Nach Saur, Allgemeines Künstlerlexikon 13 (1996) 504 (Kristiane Frank). Ein erstes Foto-Panorama aus dem Jahr 1865 erwähnt G. Hübner, Zu den Anfängen der Fotografie in der deutschsprachigen Klassischen Archäologie, IstMitt 54, 2004, 88.

${ }^{25}$ Oettermann (Anm. 2) 256f. Abb. 185. Als weitere Ruinenstätten wären zu nennen: Baalbek 1836 und Jerusalem 1835. Oettermann (Anm. 2) 257 Abb. 186.

${ }^{26}$ R. Lepsius, Denkmäler aus Ägypten und Äthiopien. Erste Abtheilung. Topographie und Architektur (Berlin 1849-1858) Taf. 15-18. Die aquarellierten Vorzeichnungen, nach denen die vier Blätter lithografiert wurden, stammen nach den Angaben auf den Blättern selbst von Johann Jakob Frey aus Basel (1813-1865), einem der drei Maler in der Expedition von Lepsius. A. von Specht (Hrsg.), Lepsius - Die deutsche Expedition an den Nil. Ausstellungskatalog Kairo (Kairo 2006) 152-157 mit einem vergleichbaren Panorama von der "größten Pyramide“. Dort wird allerdings Joseph Bonomi (1796-1878) als Autor des Panoramas bezeichnet; vgl. dazu auch $<$ http://aaew.bbaw.de/dateien/weitere archive/lepsius-archiv> (01. 10. 2008).

27 Zusammengesetzt entsprechen diese Bilder den heute beliebten fotografischen Panoramen. Digital können die Blätter mittlerweile leicht verbunden werden. Hier kann die Geschichte der archäologischen Panorama-Fotografie nicht verfolgt werden. Zu den ersten, seit den 1840er Jahren eigens konstruierten Panorama-Kameras: Meers (Anm. 8) 24. 




Abb. 4 Ferdinand Stademann, Drei Blätter aus dem „Panorama von Athen. An Ort und Stelle aufgenommen und herausgegeben von Ferdinand Stademann" (München 1841)

Die bisher besprochenen Panoramen zeigen die Ruinen wie sie sind. Eine Rekonstruktion ist nicht intendiert und wohl auch dem einzelnen Zeichner gar nicht möglich. Die antiken Stätten ordnen sich damit in das allgemeine Programm der Schausteller mit Reisebildern und Sensationen ein. Vor diesem Hintergrund gilt es, das Rom-Panorama von Josef Bühlmann und Alexander von Wagner zu analysieren und zu bewerten. ${ }^{28}$ Zunächst soll aber kurz die Geschichte seiner Entstehung skizziert und eine Charakterisierung der wesentlichen Protagonisten vorgenommen werden.

Die zu Beginn der 1880er Jahre gegründete „Panorama-Gesellschaft in München“, eine Filiale der damals den europäischen Markt beherrschenden belgischen Panorama-Gesellschaft, beauftragte 1885 den Münchner Professor Josef Bühlmann damit, für ihr Ausstellungsgebäude in der Theresienstraße Nr. 8 ein Panorama des

${ }^{28}$ Oettermann (Anm. 2) 191f. 200. 204; Sehsucht (Anm. 2) 172f. Nr. II.102f.; G. Forster, Die rekonstruierenden Bildwelten des Kunstdidaktikers, Architekturzeichners und Architekten Josef Bühlmann (Diss. München 1999, Berg 1999) 50-85 und passim; W. Schäche, Josef Bühlmann und Alexander von Wagner - die Schöpfer des Rom-Panoramas von 1888, in: Y. Asisi (Hrsg.), Rom CCCXII (Leipzig 2006) 15-26. 


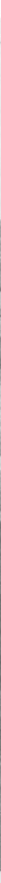

antiken Rom anzufertigen. ${ }^{29}$ Bühlmann war damals außerordentlicher Professor am Münchner Polytechnikum. Im Sommer 1886 wurde auch der Akademieprofessor Alexander von Wagner hinzugezogen. Als Vorarbeiten, die aus dieser Zeit stammen müssen, blieben quadrierte Lichtpausen - noch ohne menschliche Figuren - erhalten (Taf. 9). Deren transparente Vorlagen müssen für das Auftragen der Komposition mittels Projektion (?) auf die große Leinwand gedient haben. ${ }^{30}$ Am 2. Juli 1888, ein Jahr früher als geplant, konnte das „Rundbild vom alten Rom“ erstmals besichtigt werden, allerdings nicht ganz fertiggestellt. Die Eile wird dadurch begründet gewesen sein, dass gleichzeitig in München eine Internationale Kunstausstellung und die Deutsche Kunstindustrie-Ausstellung zahlreiche Besucher anlockten. Am 5. Mai

${ }^{29}$ Oettermann (Anm. 2) 200 lokalisiert das Panorama versehentlich in einem zweiten, erst 1885 in der Goethestraße errichteten Ausstellungsbau. Dort war aber damals noch Bruno Piglheims Panorama der Kreuzigung Christi zu sehen (Mai 1886 bis Anfang 1889). Richtig zu den zwei Münchner Panorama-Gesellschaften Oettermann (Anm. 2) 216f. Zuletzt F. Schiermeier, Panorama München, Illusion und Wirklichkeit. München als Zentrum der Panoramaherstellung (München 2009) 34-37. 60-61.

${ }^{30}$ Blaupausen von $40 \mathrm{~cm}$ Höhe und unterschiedlicher Breite. Museum für Architektur der TU München. Abbildungen der drei Pausen bei Schäche (Anm. 28) 18-19. Die Blätter sind intensiv benutzt worden und an den Rändern ausgefranst. 
1889 kam es deshalb in Anwesenheit der bayerischen königlichen Hoheiten zu einer erneuten feierlichen Eröffnung. Vom Februar 1890 bis zum Dezember 1891 war das Werk auch im Berliner ,National-Panorama' ausgestellt, um danach wieder in die Theresienstraße zurückzukehren. ${ }^{31}$ Dort blieb es bis in die späten 90er Jahre. Heute ist es verschollen, wahrscheinlich durch Gebrauch und wiederholten Transport zerstört. ${ }^{32}$ Die Presse begleitete die Ausstellung sowohl in München wie in Berlin ausführlich und begeistert. ${ }^{33}$ Außerdem blieben unterschiedlich ausführliche, zum Teil zweisprachige Beschreibungen des Panoramas erhalten, die üblicherweise durch eine vereinfachte, erläuternde Umzeichnung des Bildes ergänzt wurden und zusammen mit dem Eintrittsbillet erworben werden konnten. ${ }^{34}$ Zusätzlich edierte die Gesellschaft auch eine fotografische Reproduktion in der Art eines Leporello in zwei unterschiedlich großen Fassungen, die weite Verbreitung fanden und große Wirkung zeitigten (Faltblatt 1). ${ }^{35}$ Wirtschaftlich war das Panorama ein Erfolg: 1894 hatte die Gesellschaft in einer viermonatigen Saison dank ca. 20000 Besuchern 17000 Mark an Eintrittsgeldern eingespielt, dazu 3000 Mark durch den Verkauf der Publikationen erlöst. Nach Abzug der Unkosten warf das Bild damit 11000 Mark an Gewinn ab. ${ }^{36}$

Neben der Panorama-Gesellschaft, die den Auftrag vergab und finanzierte, waren drei Männer für das Aussehen und den Erfolg des Panoramas entscheidend: Josef Bühlmann als Entwerfer und Architekturzeichner für den Inhalt und die technisch richtige Ausführung; Alexander von Wagner für das Kolorit und die Darstellung der

31 Oettermann (Anm. 2) 204.

${ }^{32}$ In den Texten der Ausstellung im Panometer in Leipzig wurde vermutet, dass das Bild zuletzt in Boston ausgestellt wurde.

33 Presseberichte für München abgebildet bei Schäche (Anm. 28) 26. Für Berlin: Oettermann (Anm. 2) 292 Anm. 50.

${ }^{34}$ Folgende Texte konnte ich einsehen: Anonym [Franz von Reber], Rom mit dem Triumphzuge Constantins im Jahre 312. Rundgemälde von Prof. J. Bühlmann und Prof. Alex. Wagner. Mit einer Orientierungstafel (München 1888) (35 S., Plan, Falttafel mit Umzeichnung des Panoramas und Benennung der Bauten); F. von Reber, Übersichtliche Erklärung des Rundbildes von Rom mit dem Triumphzuge Constantins 312 n. Chr. Beilage zum ausführlichen Führer (München 1888) (7 S.); Kurzer Führer zum Rundbild von Rom 312 n. Chr. Zur schnelleren Orientierung nach Prof. Dr. Franz von Rebers ausführlichem Werk zusammengestellt (gleichzeitig Französisch), (München 1888) (15 S. zweispaltig dt./frz.). - Diese Publikationen beziehen sich auf eine „ausführliche“ Fassung: F. von Reber, Rom mit dem Triumphzuge Konstantins im Jahre 312: Rundgemälde von Prof. J. Bühlmann und Prof. Alex. Wagner [Mit einer Orientierungstafel, der Skizze des Panoramas und 23 Textillustrationen], beschrieben von Franz von Reber (München 1888, $\left.{ }^{2} 1889\right)$ 6. 160 S., 2 Tafeln, 1 Plan.

35 Das alte Rom mit dem Triumphzuge Kaiser Constantin's im Jahre 312 n. Chr. Rundgemälde von den Professoren J. Bühlmann und Alexander von Wagner in München (München 1890). Der genaue Titel und seine Gestaltung variieren bei den mir bekannten Exemplaren. Verbreitet erhalten ist eine ,kleine' Fassung (aufgefaltet ca. $40 \times 100 \mathrm{~cm})$. Die, große' $(80 \times 200 \mathrm{~cm})$ Edition bildet Forster (Anm. 28) 53 Abb. 26 ab.

${ }^{36}$ Bilanz abgedruckt bei Oettermann (Anm. 2) $191 \mathrm{f}$. 
Figuren und schließlich Franz von Reber als Berater und Autor der beschreibenden Publikationen. In unserem Zusammenhang sind vor allem die Anteile von Bühlmann und von Reber von Bedeutung.

Die zentrale Figur war zweifelsfrei Josef Bühlmann (1844-1921). ${ }^{37}$ Als Schweizer an der Akademie in München in den Jahren 1863 bis 1872 ausgebildet, kehrte er zunächst nach Luzern zurück. Schon 1875 kam er jedoch erneut nach München und erhielt 1878 am Polytechnikum, der heutigen Technischen Universität, eine außerordentliche Professur für ,Bauformenlehre und Decorativen Ausbau', die 1888 zu einem Lehrstuhl für Bauzeichnen angehoben wurde. ${ }^{38} \mathrm{Ab} 1908$ lehrte er in der Nachfolge von August Thiersch auch ,Antike Baukunst'. Als Architekt blieb Bühlmann wenig erfolgreich: Die einzige von ihm - zusammen mit anderen - konzipierte, ausgeführte und noch erhaltene Arbeit scheint der Friedensengel auf dem Isarhochufer zu sein. Seinen eigentlichen Erfolg machten dagegen wissenschaftliche und didaktische Publikationen aus: Eine dreiteilige „Architektur des klassischen Alterthums und der Renaissance "39 erschien ab 1872 in mehreren Auflagen und im Handbuch der Architektur verfasste er verschiedene Abschnitte, um nur das Wichtigste zu nennen. ${ }^{40}$ Dazu kommen in unserem Kontext kleinere, meist erst nach dem Panorama erschienene Schriften: „Der römische Tempelbau“, ${ }^{41}$ „Das Mausoleum von Halikarnaß“, ${ }^{42}$ „Der Palast der Flavier auf dem Palatin in Rom“ ${ }^{43}$ Es handelt sich dabei stets um Rekonstruktionsversuche mit wissenschaftlichem Anspruch. Vergleichbare Zeichnungen steuerte er auch als Illustrationen für andere Werke bei, so für das damals als Prachtwerk gelobte „Hellas und Rom“ von Jakob von Falke. ${ }^{44}$ Eine Zeich-

37 Zu Bühlmanns Leben: Thieme-Becker Bd. 14 (1921) 473f. (Max Baumgärtel); Saur, Allgemeines Künstlerlexikon 15 (1997) 37f. (Verena Karnapp; Martin Ziermann); W. Nerdinger, Die Architekturzeichnung (München 1986) 128-137; ders., Architekturschule München 1868-1993 (München 1993) 59. 172. Die Dissertation von Forster 1999 im Fach Kunstpädagogik (Anm. 28) enthält einige wichtige Dokumente und bildet vor allem die meisten Blätter und Arbeiten Bühlmanns ab, die im Architekturmuseum der TU München verwahrt werden. Wegen ihres chaotischen Aufbaus und der wissenschaftlich unzulänglichen Vorgehensweise bleibt sie jedoch ein unerfreulicher Lesestoff. Forster druckt verschiedene Nachrufe ab: S. 31. 32-35 und nach S. 301 (unpaginiert). - Teile des Nachlasses von Bühlmann liegen im Architekturmuseum der TU München.

${ }^{38}$ Die Denominationen von Bühlmanns Aufgaben schwanken in der mir bekannten Literatur.

39 J. Bühlmann, Die Architektur des klassischen Alterthums und der Renaissance, 3 Teile (Stuttgart 1872/86) und weitere Auflagen.

40 J. Bühlmann, Die Bauformenlehre. Handbuch der Architektur I, 2 (Darmstadt 1896).

${ }^{41}$ J. Bühlmann, Der römische Tempelbau. Die Baukunst, Ser. 2, H. 9 (Berlin 1902).

42 J. Bühlmann, Das Mausoleum von Halikarnaß, Zeitschrift für Geschichte der Architektur 2, H. 1, 1908/09, 1-25.

${ }^{43}$ J. Bühlmann, Der Palast der Flavier auf dem Palatin in Rom, Zeitschrift für Geschichte der Architektur 1, 1907/08, 113-134.

${ }^{44}$ J. von Falke, Hellas und Rom (Stuttgart 1878-80). Abbildungen aus diesem Werk finden sich bis in jüngste Gesamtdarstellungen der antiken Architektur: W. Lübke, Geschichte der Archi- 
nung soll noch besonders hervorgehoben werden, da sie in mancher Hinsicht einen Vorläufer des Panoramas bildet (Abb. 5). Das 1879 datierte Blatt zeigt „Rom zur Zeit des Kaisers Aurelianus". ${ }^{45}$ Bühlmann war also bereits 1885 ein erfahrener Rekonstruktionszeichner, der seine Kenntnis römischer Topografie und Architektur auf ganz verschiedenen Ebenen mehrfach bewiesen hatte. Er beherrschte außerdem schwierigste perspektivische Techniken. Damit brachte er die besten Voraussetzungen für den Entwurf und die Anfertigung eines Panoramas mit.

Auch Franz von Reber (1834-1919) war als gelehrter Interpret am Erfolg und der Wirkung des Panoramas beteiligt. Er war ab 1863 der erste Professor für Kunstgeschichte am Polytechnikum in München und damit ein Kollege Bühlmanns. Von 1875 bis 1907 leitete er die Central-Galeriedirection, nahm also eine einflussreiche Stellung im Münchner Kunstbetrieb ein. Zu seinen vielfältigen Schriften gehören Kataloge der Pinakothek und populäre Bildbände im Bruckmann-Verlag. ${ }^{46}$ Ein Schwerpunkt seiner Arbeit galt jedoch der antiken Kunst, Topografie und Architektur. 1858 wurde er mit einer Schrift zur "Lage der Curia Hostilia und der Curia Julia“ in München habilitiert. ${ }^{47} 1863$ und erneut 1879 erschien ein anspruchsvoller Führer mit dem Titel „Die Ruinen Roms und der Campagna“.48 1866 verfasste er eine „Geschichte der Baukunst im Alterthum“, und seine „Kunstgeschichte des Alterthums“ von 1871 wurde sogar ins Englische übertragen. ${ }^{49}$ Eine Vitruv-Übersetzung ist erst jüngst als Nachdruck wieder in den Handel gelangt. ${ }^{50}$ Aus späteren Jahren stammen verschiedene Akademieschriften, in denen sich von Reber vor allem der frühen griechischen, der hethitischen sowie der phrygischen Architektur zuwandte. Bei diesen Themen ist wieder eine Zusammenarbeit mit Bühlmann konkret belegt, der für eine dieser Schriften das Megaron im Palast von Tiryns 1896 zeichnerisch rekonstru-

tektur I ${ }^{6}$ (Leipzig 1884) 304 Abb. 285 (Trajansforum); 327 Abb. 306 (Röm. Wohnhaus); M. Grassnick - H. Hofrichter (Hrsg.), Materialien zur Baugeschichte I (Braunschweig 1982) Nr. A. 142 (Forum Romanum und Capitol); W. Koch, Baustilkunde (München 1988) 30 (ebenso).

45 Datiert und signiert 1879. Die historische Druckplatte ist erhalten $(42,6 \times 85,6 \mathrm{~cm})$. Sie wurde 1961 von dem Drucker Hans Rischert restauriert. Neue Abzüge liegen im Archiv des Architekturmuseums der TU München. Die Vogelschau nahm eine Doppelseite in von Falke (Anm. 44) zwischen den S. 220 und 221 ein.

46 F. von Reber - A. Bayersdorfer (Hrsg.), Klassischer Bilderschatz, 12 Bde. (München 18891900); F. von Reber, Klassischer Skulpturenschatz, 4 Bde. (München 1897-1900).

47 F. von Reber, Die Lage der Curia Hostilia und der Curia Julia (München 1858) 59 S.

48 F. von Reber, Die Ruinen Roms und der Campagna (Leipzig 1863, ${ }^{21879)}$ ). Ein leicht überarbeiteter und aktualisierter Nachdruck erschien in Kettwig 1991. Außerdem: Album der Ruinen von Rom mit Einleitung und Tafelerklärung von F. Reber (Leipzig 1883). Das Frontispiz zeigt eine Rekonstruktion des Forum Romanum nach S von Bühlmann 1883.

${ }^{49}$ F. von Reber, Geschichte der Baukunst im Alterthum. Nach den Ergebnissen der neueren wissenschaftlichen Expeditionen bearbeitet, mit 274 Holzstichen im Text (Leipzig 1866); F. von Reber, Kunstgeschichte des Alterthums (Leipzig 1871; engl. London 1882).

50 Vitruv, De Architectura libri decem (Stuttgart 1865); Nachdruck Wiesbaden 2004. 


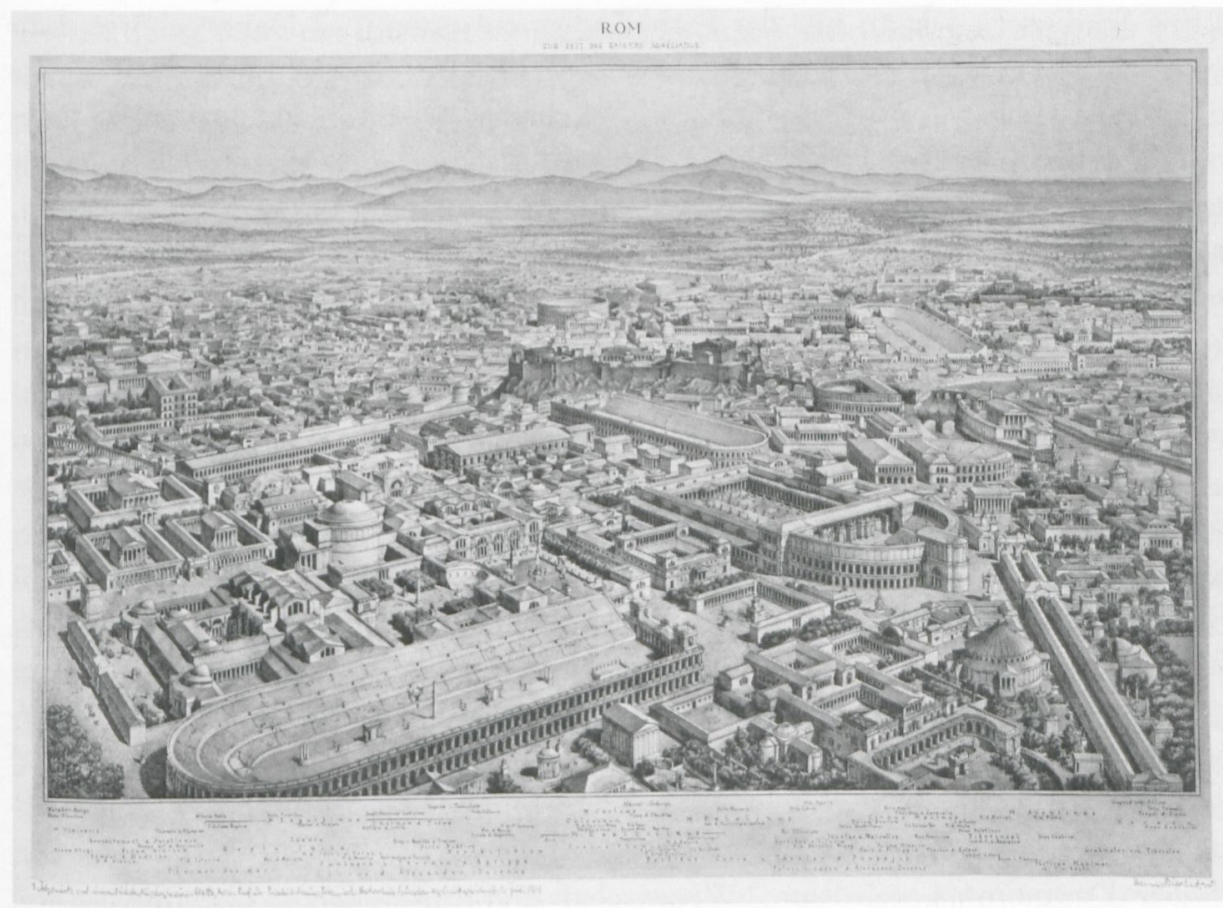

Abb. 5 Josef Bühlmann, Rom zur Zeit des Kaisers Aurelianus, 1879. Architekturmuseum der TU München

ierte. ${ }^{51}$ von Reber war damit gleichfalls ein ausgewiesener und einflussreicher Kenner der antiken Architektur im Allgemeinen und Roms und seiner Topografie im Besonderen. Von ihm stammen die oben genannten unterschiedlichen Beschreibungen des Panoramas, die man an Ort und Stelle erwerben konnte. Seine Texte enthalten zunächst eine Schilderung der historischen Ereignisse um die Schlacht an der Milvischen Brücke und dann eine Beschreibung der einzelnen Bauten, deren Geschichte und ihrer Funktionen. In einem Beitrag zum ersten Band einer neuen Kunstzeitschrift nimmt er zu dem Panorama auch wertend Stellung. ${ }^{52}$ Der schwierigen Aufgabe sei man nicht „blos mit Kunst, sondern auch, und zwar vor Allem, mit Wissenschaft näher (ge)treten (...) Der Ausgangspunkt ist der der wissenschaftlichen Reconstruction. Die Gründlichkeit und Tiefe der dazu herangezogenen topografi-

51 F. von Reber, Ueber das Verhältnis des mykenischen zum dorischen Baustil, Abhandlungen der III. Classe der k. Akademie d. Wissenschaften 21, 3. Abt., 1898, Taf. (1) und (2). - Der Palast war 1884/85 von Heinrich Schliemann und Wilhelm Dörpfeld ausgegraben und 1886 publiziert worden.

${ }^{52}$ F. von Reber, Das Rundbild der Stadt Rom von Professor Jos. Bühlmann und Professor Alex. Wagner, in: H. E. von Berlepsch-Valendàs (Hrsg.), Die Kunst unserer Zeit 1, 1889, 1-10. 
schen Studien (...) kann nur der Fachkundige ermessen (...) Dann gehörte dazu vollendete Kenntnis der römisch-classischen Architecturformen (...) Endlich mußte in perspectivischer Construction Ungewöhnliches geleistet werden. "Das antike Rom bot dabei besondere Schwierigkeiten: „Dadurch, daß von allen Hauptstädten des Alterthums keine so weit in's Detail ihrer antiken Gestaltung bekannt ist, wie Rom, wurde die Sache nur um so schwieriger gemacht, indem der Fantasie nur sehr beschränkte Stellen geboten, der Fesseln aber unzählige erwachsen waren. Freilich konnte dadurch das Werk nur um so gediegener und von einer sachlichen Wahrheit werden, wie sie bisher auf dem Gebiete der Totalrestauration eines antiken Schauplatzes noch nicht erreicht worden ist. "53 Gegen Ende meldet er aber auch Kritik an. „Der Glaube an die absolute Wahrheit des Dargestellten“ werde dadurch beeinträchtigt, daß die Rekonstruktionen zu geleckt erschienen und keine pittoreske Patina besäßen. ${ }^{54}$ Schließlich referiert von Reber das Schreiben eines weiteren hervorragenden Romkenners, Ferdinand Gregorovius (1821-1891), der damals meist in München lebte und das Panorama daher sicher aus eigener Anschauung gut kannte. Auch Gregorovius lobt die Wissenschaftlichkeit des Werkes. Er betont aber noch einen weiteren Aspekt: „Indem Architekt und Maler infolge des gewählten Standpunktes darauf verzichten mußten, die ungeheuren Massen des bürgerlichen Stadtkörpers auszubreiten, haben sie im wesentlichen und wirkungsvoll dargestellt, was die Hauptsache sein mußte: Das monumentale Rom. "Wissenschaft und Kunst seien, so Gregorovius, wie nicht oft, eine glückliche Verbindung eingegangen. ${ }^{55}$ Es muss erstaunen, dass gerade die mehrfach beschworene Wissenschaftlichkeit von Bühlmanns Rekonstruktionen schon bald in Zweifel gezogen wurde. In einem grundsätzlich positiv gestimmten Nachruf heißt es 1921: „[Bühlmann] schuf (...) das herrlich, einzig dastehende Panorama: Das Alte Rom (...), welches allerdings mehr ein geschichtliches Demonstrationswerk, als eine auf wissenschaftlich-topographischen Grundlagen aufgebaute Rekonstruktion ist." 56

Es ist hier nicht der Platz, das riesige Panorama im Einzelnen zu durchforsten und seine Richtigkeit oder Plausibilität im Vergleich mit unserem Kenntnisstand zu überprüfen. ${ }^{57}$ Einige Punkte sollen jedoch kurz angesprochen werden, um die Arbeitsweise Bühlmanns zu charakterisieren. Besonders, fehlerhaft' erweist sich das Panorama natürlich im Bereich der Kaiserfora und dort vor allem am Trajansforum wie am Forum Pacis, beides Bereiche der antiken Stadt, die damals nur in geringem Umfang ausgegraben waren. Unrichtig ist auch die Lokalisierung des Circus Flaminius, der zu sehr an das Capitol herangerückt wurde. Bei anderen Bauten, deren Aussehen ebenfalls gar nicht oder wenig bekannt war, nutzte Bühlmann seine Freiheit, um ihre

\footnotetext{
53 von Reber (Anm. 52) 6.

54 von Reber (Anm. 52) 9.

55 von Reber (Anm. 52) 9-10. Brief an Bühlmann vom 8. 7. 1888.

56 Luzerner Tagblatt vom 7. 11. 1921, nach: Forster (Anm. 28).

57 Vgl. dazu auch die Bemerkungen hier im Beitrag Cain.
} 
Gestalt dem Bedarf der historischen Handlung anzupassen. So legte er vor das Tabularium eine Halle, um dort die Christen als Beobachter des heidnischen Zuges zu postieren. Gegenüber wurde der Tempel der Iuno Moneta genau so angeordnet, dass Raum für eine große Opferzeremonie blieb.

Andere Stellen belegen dagegen eine ausgezeichnete Kenntnis der historischen Quellen: So wird in von Rebers Beschreibung eigens auf die Hütte des Romulus auf dem Palatin hingewiesen, die im Bild nur sehr klein angedeutet wurde. Auch der Anbau an der Rückseite des Capitoltempels zeugt vom Studium der literarischen Quellen. Die Erläuterungen von Rebers benennen ihn als aedes thensarum, ein Gebäude zur Aufbewahrung der für kultische Zwecke genutzten zweirädrigen Wagen, der thensae, dessen genaue Lokalisierung bis heute nicht gesichert ist. ${ }^{58}$ Die Rekonstruktion des Veiovis-Tempels - in der Senke zwischen Arx und Capitol - in Form eines Rundbaus mit Schuppendach und Oculus geht dagegen auf die falsche Interpretation eines Passus bei Vitruv (IV. 8. 4) zurück. Der Bau wird dort zwar im Zusammenhang mit Rundtempeln erwähnt, das Tertium des Vergleichs betrifft jedoch nur die Anlage der Vorhallen, nicht aber die Gestalt des ganzen Tempels. ${ }^{59}$ Ebenfalls als Nachweis gelehrter Belesenheit muss die kleine Öffnung verstanden werden, die im Dach des Iuppiter-Capitolinus-Tempels zu beobachten ist. Sie weist, wie Paolo Liverani während des Kolloquiums überzeugend anmerkte, offenbar auf ein kleines Heiligtum des Gottes Terminus hin, das sich innerhalb des Tempels befand. Da dessen Altar aus rituellen Gründen unter freiem Himmel, sub divo, liegen musste, blieb der Tempel an dieser Stelle ohne Abdeckung. ${ }^{60}$

Wie zu erwarten, spiegelt sich schließlich vor allem in den frei ergänzten Partien die Zeitgebundenheit des Entwerfers. So wirken die archäologisch nicht belegten ,Propyläen' des Iuppiter-Tempels eher wie Pavillons eines Ausstellungsgeländes des späten 19. Jahrhunderts. Das große Reiterdenkmal links des Aufgangs, ein - gleichfalls nicht antik überliefertes - Monumentum Iuliorum, trägt bereits in der Idee seiner Erfindung, aber auch in seiner kruden Mischung von Motiven des Mausoleums von Halikarnass mehr wilhelminische als antike Züge und hätte bei den damals häufigen Wettbewerben zu monumentalen Denkmälern sicher eine gute Figur gemacht. Die zahlreichen, mit Victorien besetzten Säulen im Umkreis des capitolinischen Tempels ähneln der bereits genannten Friedenssäule Josef Bühlmanns in München.

58 Zur aedes thensarum: LTUR I (Rom 1993) 17 (Giuseppina Pisani Sartorio).

${ }^{59}$ Der (rechteckige) Veiovis-Tempel wurde erst in den dreißiger Jahren unter dem Senatorenpalast freigelegt. Vgl. zuletzt LTUR V (Rom 1999) 99-100 (Margherita Albertoni).

${ }^{60}$ Zum Terminus-Altar: LTUR V (Rom 1999) 27-28 (Gianluca Tagliamonte). Zuerst hatte ich gedacht, dass die Dachöffnung mit der im 19. Jahrhundert verbreiteten Diskussion um hypäthrale Tempel stünde. In einem Beitrag hatte von Reber selbst zu der Frage Stellung bezogen und die Rekonstruktion solcher Tempel abgelehnt. Die im Panorama angedeutete Dachöffnung wäre für eine solche Deutung deutlich zu klein. F. von Reber, Die Hypäthralfrage, Repertorium für Kunstwissenschaft 3, 1880, 61-65. 
Man greift jedoch zu kurz, wenn man das Panorama nur als topografisch-bauhistorische Studie versteht. Es handelt sich, wie eingangs schon gesagt, um ein gewaltiges Historienbild. Dies wird auch bei Bühlmanns Anteil deutlich. Hatte er für seine Vogelschau mit der Regierungszeit des Aurelian und der damals entstandenen großen Stadtmauer noch einen vor allem baugeschichtlich sinnvollen Moment gewählt, so ist das Jahr 312 ereignisgeschichtlich von besonderer Bedeutung: Es steht symbolisch für den Sieg des Christentums. Die Wahl des Standortes ist damit eindeutig dem Thema geschuldet. Rekonstruktionen des Forum Romanum und des dahinter liegenden Capitols hatte es schon mehrfach gegeben, doch der Standort auf der östlichen Erhebung dieses Hügels war neu und für den geschichtsmächtigen Moment der Begegnung von Heidentum und Christentum besonders geeignet. Für den Bauforscher bot dieser Blickwinkel jedoch besondere Schwierigkeiten, da die Topografie des Capitols wegen dessen fast vollständiger Überbauung kaum bekannt war. Bewusst als Momentaufnahme wird von Bühlmann außerdem im Hintergrund des Forums ein eingerüstetes, monumentales Gebäude gezeigt (Abb. 6). Es ist die Maxentiusbasilika, die sich damals noch im Bau befand und unter Konstantin umgestaltet, umbenannt und fertiggestellt werden sollte. Solche Details dürften jedoch nur dem Kenner aufgefallen sein. Die eigentliche historische Verortung leisteten dagegen die Figurenszenen Alexander von Wagners (1844-1921). Der aus Ungarn stammende Maler war als Schüler Pilotys in München zu einer Professur gekommen. Heute weitgehend vergessen, besaß er damals ein gewisses Renommée als Maler von ungarischen und spanischen ländlichen Szenen, ebenso wie von Historienbildern. Das für das Panorama gelobte Kolorit kann man heute nicht mehr nachvollziehen. In der Ikonografie einzelner Figuren finden sich aber immer wieder Hinweise auf seine Kenntnis der antiken Skulptur: So sitzt die Kaiserin auf einem Stuhl, wie die damals noch so genannte Sitzstatue der Helena (Mutter Konstantins!) in den Kapitolinischen Museen ${ }^{61}$ während er für die Opferszene vor dem Iuno-Moneta-Tempel auf antike SuovetauriliaDarstellungen zurückgriff. Insgesamt gelingt es Wagner, eine Stimmung zu erzeugen, die einem kolossalen ,Sandalenfilm' nicht unähnlich ist. ${ }^{62}$

Unter den Stadtrekonstruktionen des 19. Jahrhunderts kann Bühlmanns Panorama durch seinen Anspruch und seinen Umfang eine Sonderstellung beanspruchen. Zwar waren seit circa 1810 sowohl Athen wie auch das Forum Romanum mit dem angrenzenden Capitolshügel gern zeichnerisch wiederhergestellt worden, doch beschränkten sich solche Arbeiten stets auf ein handliches Blattformat und mussten außerdem die Stadtlandschaften mangels archäologischer Befunde weitgehend hypothetisch auffüllen. ${ }^{63}$ In der Größe konnte nur ein Halb-Panorama von Pergamon

${ }^{61}$ W. Helbig, Führer durch die öffentlichen Sammlungen klassischer Altertümer in Rom II ${ }^{4}$ (Tübingen 1966) 153f. Nr. 1326 (H. von Steuben).

62 von Reber (Anm. 52) bildet in diesem Aufsatz zahlreiche Vorstudien Wagners ab.

63 Zum Forum kenntnisreich und mit vielen Abbildungen F. Salmon, Building on Ruins (Aldershot 2000) $98 \mathrm{ff}$.; ders., Perspectival Restoration Drawings in Rome, AntJ 83, 2003, 397-424. 


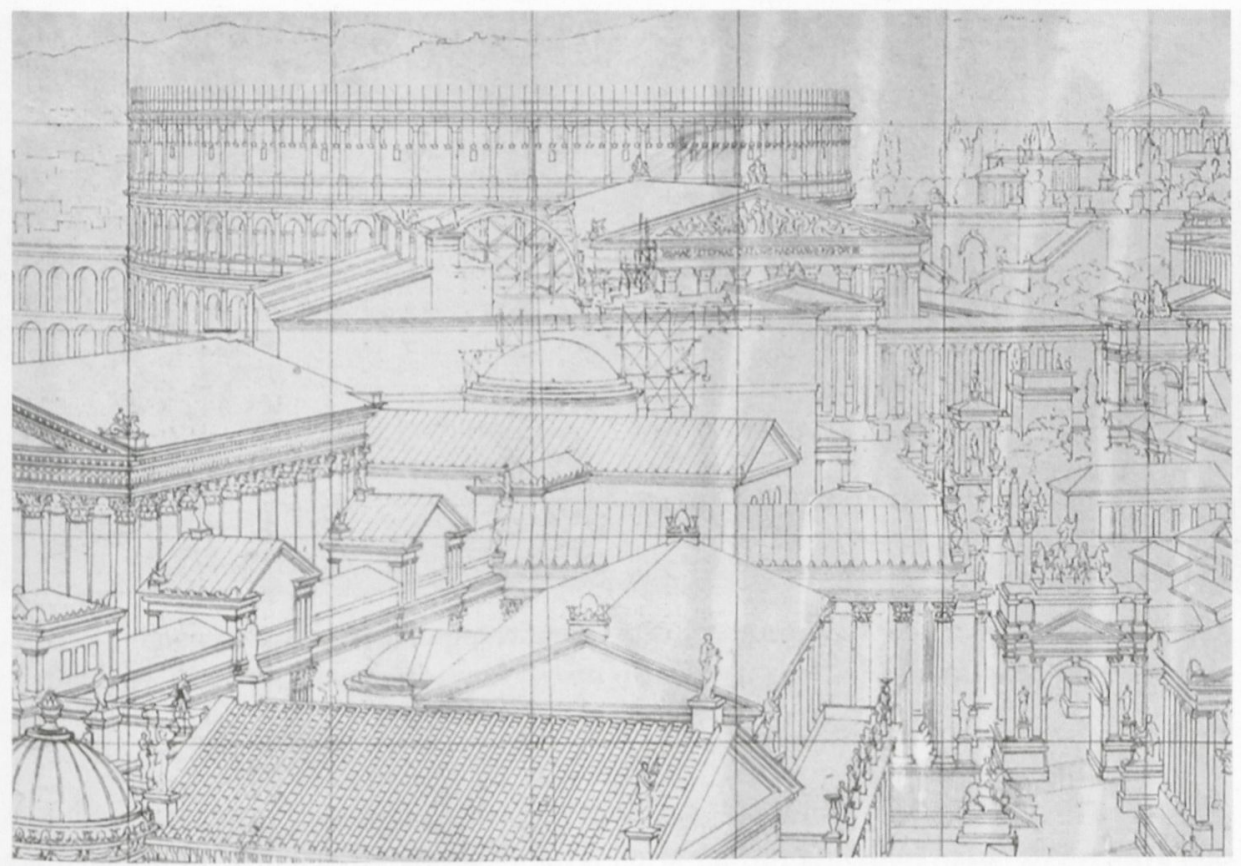

Abb. 6 Josef Bühlmann, Lichtpause zur Vorbereitung des Rompanoramas mit Darstellung der Maxentius-Basilica (Ausschnitt). Architekturmuseum der TU München

konkurrieren, das 1886 bei der Ausstellung zum 100jährigen Jubiläum der Akademie der Künste in Berlin gezeigt wurde (Abb. 7). ${ }^{64}$ Die Maler Alexander Kips (1858-1910) und Max Friedrich Koch (1859-1930) hatten sich zu Vorstudien 1885 im deutschen Grabungshaus am Ort aufgehalten und genossen bei ihrer Arbeit an der $14 \mathrm{~m}$ hohen und $65 \mathrm{~m}$ langen Leinwand die Unterstützung des Grabungsarchitekten Richard Bohn. Das Schaubild feierte ebenso wie eine Rekonstruktion des großen Zeusaltares und ein Nachbau der Ostfassade des Zeustempels in Olympia (beide im Maßstab 1:1) die Leistungen der deutschen Archäologie. In Berlin war man begeistert, auch unter Archäologen und Bauforschern. Das Panorama habe „mehr als irgendetwas anderes dazu beigetragen (...) weiten Kreisen eine richtige Idee der alten pergamenischen Herrlichkeit zu geben“, konstatierten später die Biografen des Ent-

Außerdem V. Kockel, Stadtvisionen, in: U. Hassler - W. Nerdinger (Hrsg.), Das Prinzip Rekonstruktion (Zürich 2010) 124-143.

${ }^{64}$ Jetzt ausführlich U. Wulf, „Die erfreulichsten Spuren hatten hinterlassen die beiden Maler Kips und Koch ...". Das Pergamon-Panorama der Jubiläumsausstellung 1886, IstMitt 54, 2004, 113-127 mit Beil. 2. Der Begleitband zur Jubiläumsausstellung stammte von Ernst Fabricius und Ludwig Pietsch, Führer durch das Pergamon-Panorama sowie durch das Kaiser-Diorama der centralafrikanischen Erforschungs-Expedition ${ }^{2}$ (Berlin 1886). 




Abb. 7 Alexander Kips und Max Friedrich Koch, Orientierungsskizze für das PergamonHalbpanorama. Aus: Führer durch das Pergamon- und Olympia-Panorama (Berlin 1886). Falttafel

deckers von Pergamon, Carl Humann. ${ }^{65}$ Der kleine begleitende Führer bescheinigte den Künstlern, „das Kolossalbild der alten Attalidenstadt zugleich in so hoher poetisch-malerischer Schönheit, überzeugender Wahrheit und, zum großen Teil wenigstens, genau nachweisbarer Richtigkeit" gestaltet zu haben. ${ }^{66} \mathrm{Im}$ archäologischen Anspruch unterschied sich das Pergamon-Panorama also in nichts von Bühlmanns Rom. Vor allem für die Oberstadt entsprach es genau den Angaben der Ausgräber, wie Ulrike Wulf im Detail gezeigt hat, und war damit noch ,wissenschaftlicher ${ }^{\text {' als }}$ dessen römische Rekonstruktionen. Dennoch merkte von Reber gegenüber dieser Konkurrenz wohl nicht ganz zu Unrecht herablassend an, es sei ,von recht tüchtiger und erfreulicher Wirkung (...), wenn auch die Beschränkung auf eine hemicyklische Darstellung der Illusionswirkung nicht eben vorteilhaft war" ${ }^{67}$ Genau darin muss der wesentliche Unterschied gelegen haben. Mit der Entscheidung, Pergamon in einem Blick aus der Distanz darzustellen, hatten Kips und Koch die Möglichkeit vergeben, den Besucher vollständig in die Antike eintauchen zu lassen, wie das im konstantinischen Rom möglich war.

Andere, ungefähr zur selben Zeit entstandene Panoramen konnten in ihrer Detailgenauigkeit nicht mit ,Pergamon' und ,Rom' konkurrieren. So bezeichnete von Reber den 1888 an der gleichen Stelle wie das Pergamon-Panorama gezeigten und ebenfalls von Max Friedrich Koch gemalten „Brand Roms im Jahr 64 n. Chr.“ abfällig als „eine Bühnendecoration (...) eine künstlerische Fata Morgana, die (...) es weder

${ }^{65}$ C. Schuchhardt - Th. Wiegand (Hrsg.), Der Entdecker von Pergamon, Carl Humann ${ }^{2}$ (Berlin 1931) 85.

66 Fabricius - Pietsch (Anm. 64) 9.

67 von Reber (Anm. 52) 4. 


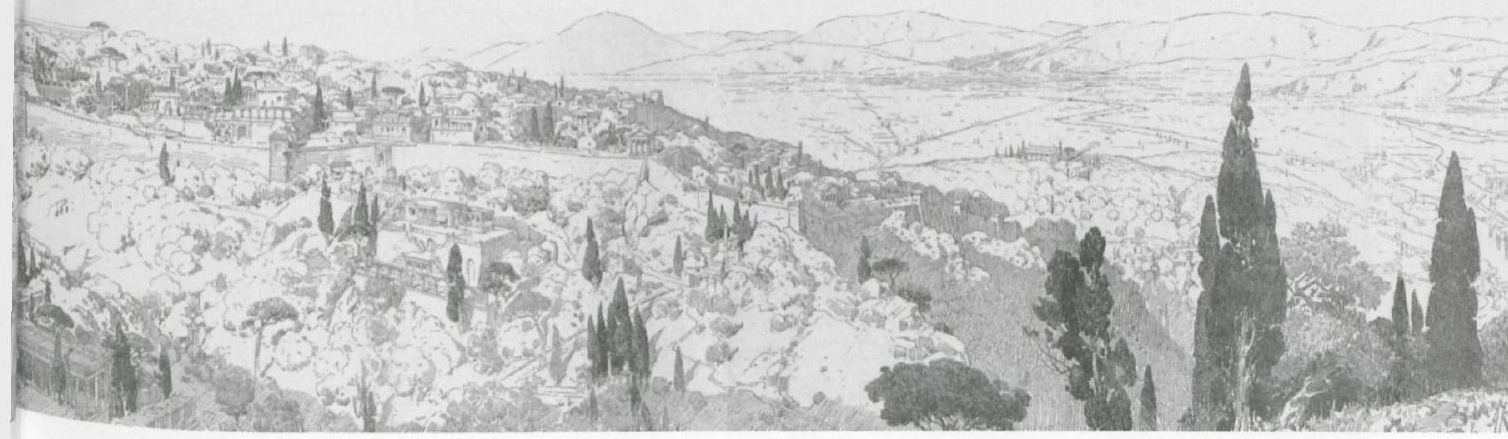

mit dem Gang des Brandes noch mit Rom genau nahm. "68 In Bruno Piglheims ungemein erfolgreicher „Kreuzigung“, die man 1886 in München hatte sehen können, war nach Meinung von Rebers die Stadt Jerusalem zu wenig in die Handlung einbezogen worden. ${ }^{69}$ Dramatisch mag das 1882 in Neapel ausgestellte Werk „Ultimi giorni di Pompei" von G. Castellani gewesen sein, von dem aber nur der Titel überliefert ist, so dass wir den Grad seiner archäologischen ,Richtigkeit' nicht beurteilen können. ${ }^{70}$ In anderen Panoramen dagegen, wie bei der bis heute erhaltenen Kreuzigungsszene von Gebhard Fugel (1863-1939) in Altötting von 1903, bemühten sich Künstler jedoch immer wieder erfolgreich um eine korrekte Rekonstruktion der antiken Architektur, auch wenn sie das Geschehen in keiner Weise so dominierte wie im Rom-Panorama. ${ }^{71}$ Bühlmann selbst hatte ebenfalls Vorarbeiten zu vergleichbaren Darstellungen von Athen und Jerusalem geleistet. Offensichtlich fanden sie aber nicht das Interesse der Investoren, um in die notwendige Größe umgesetzt zu werden. ${ }^{72}$

Mit dem Aufkommen des Films als neuem Bildmedium verloren die teuren Panoramen viel von ihrer Faszination beim großen Publikum und verschwanden allmäh-

\footnotetext{
68 von Reber (Anm. 52) 5-6; dazu auch Oettermann (Anm. 2) 204.

69 Oettermann (Anm. 2) 216-217 und Leporello 5.

70 Siehe Anm. 20.

${ }^{71}$ W. Koenigs, Die Architektur des alten Jerusalem auf dem Panorama von Altötting, in: M. Petzet (Hrsg.), Das Panorama von Altötting. Bayerisches Landesamt für Denkmalpflege, Arbeitsheft 48 (München 1990) 36-43; Oettermann (Anm. 2) 204. 274 nennt weitere Panoramen historischer Thematik, von denen mir jedoch keine Abbildungen bekannt sind.

${ }^{72}$ Deutsche Bauzeitung 48, 1914, 334-335; Forster (Anm. 28) 32-33.
} 


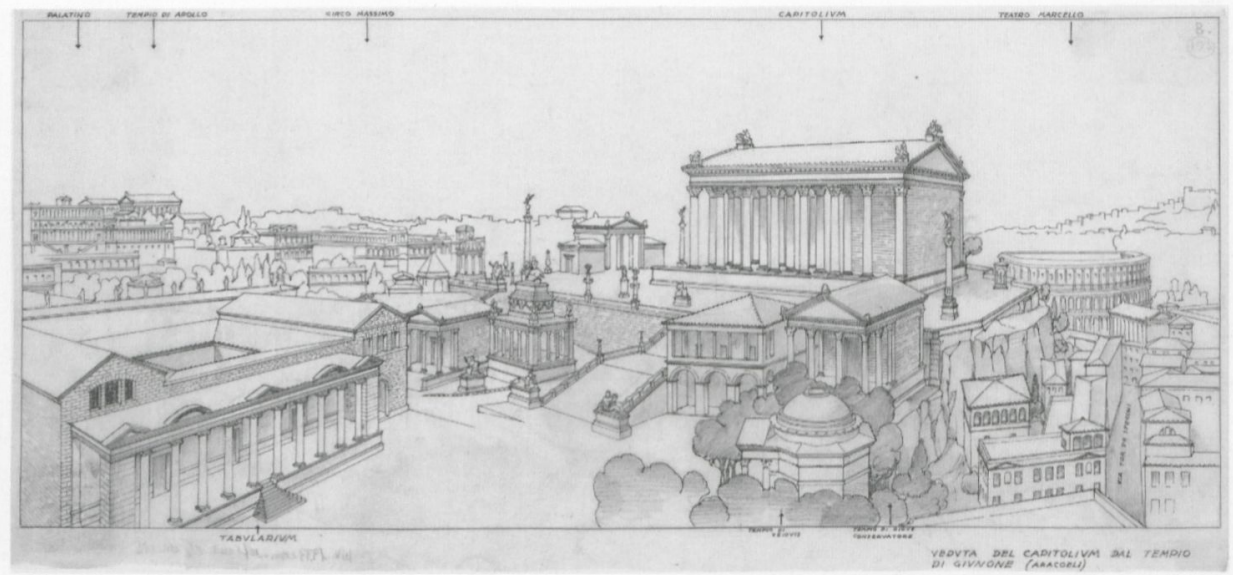

Abb. 8 Italo Gismondi, Rekonstruktion der kaiserzeitlichen Bebauung des Capitols. Soprintendenza Archeologica di Roma (ASSAR P. Altemps Inv. 1931)

lich aus der Öffentlichkeit. Das Foto-Leporello von Bühlmanns ,Rom' (Faltblatt 1) sollte aber noch einmal zu einer archäologischen Arbeit anregen. In der Folge der groBen Freilegungen im Bereich der Kaiserfora suchte der Architekt und Bauforscher Italo Gismondi nach einem geeigneten Medium, diese Ergebnisse durch Rekonstruktionen adäquat zu vermitteln. Aus der Zeit um 1930 sind drei Zeichnungen von ihm erhalten, auf denen er mit unterschiedlichen Darstellungstechniken experimentierte: Eine Vogelschau illustriert die Folge der neu ausgegrabenen Kaiserfora mit dem Colosseum im Hintergrund. Ein zweites Blatt bietet eine orthogonal projizierte Ansicht des Capitols von Südwesten. Die dritte zeigt schließlich den Capitoltempel selbst und seine Umgebung von Osten in der für ein Panorama eigentümlichen Verzerrung (Abb. 8). ${ }^{73}$ Ein Vergleich macht deutlich, dass Gismondi das entsprechende Foto aus dem Leporello Bühlmanns weitgehend durchgezeichnet hat, allerdings mit markanten Korrekturen. Unter anderem fehlen die großen Propyläen, die aedes thensarum und ein Ehrenbogen. Zwischen den Veiovis-Tempel und das Capitol sind statt dessen ein Bau mit zweigeschossiger Loggia und ein kleiner Tempel des Iuppiter Conservator eingefügt worden, hohe Wohnbauten ziehen sich entlang der felsig dargestellten Nordflanke des Capitols. Anderes bleibt: so der fälschlich kreisrunde Veiovis-Tempel und das Monumentum Iuliorum. Gismondi bediente sich also der komplizierten Konstruktionsweise Bühlmanns und korrigierte sie aufgrund seiner aktuellen Kenntnisse. Gismondi hat aber diese Abbildungsmodi nicht weiter ausgebaut, sondern

73 Dazu V. Kockel, Gismondi Panoramista?, in: F. Filippi (Hrsg.), Ricostruire l'Antico prima del virtuale. Italo Gismondi, un architetto per l'archeologia (1887-1974). Ausstellungskatalog Rom (Rom 2007) 271-273 mit Abb. auf S. 267-269. Gismondis ,Panoramablick' misst 30,5 × 95 cm, müsste damit wohl auf die ,große Edition von Bühlmann zurückgehen. 
schließlich das große Stadtmodell entworfen, das sich neuen Erkenntnissen anpassen lässt und noch heute die Besucher des Museo della Civiltà Romana in Rom wegen seiner Anschaulichkeit begeistert. ${ }^{74}$ Die Tage des archäologischen Panoramas waren endgültig vorbei. ${ }^{75}$

„Schönheit - Wahrheit - Richtigkeit“, unter diesen Kriterien bewerteten die Zeitgenossen die ,archäologischen“ Panoramen. Nicht „blos mit Kunst, sondern auch, und vor allem, mit Wissenschaft" sollten sie geschaffen werden. Sogar „die absolute Wahrheit des Dargestellten“ verlangte der Gelehrte. Dass Josef Bühlmann im Verein mit Alexander von Wagner in seinem Rom-Panorama diese Anforderungen in den Augen der Kritiker am besten erfüllte, kann man heute wieder verstehen, wenn man das Panometer in Leipzig betreten hat. Man verspürt ebenso den genuinen Zauber eines Rundgemäldes: Man fühlt sich mitten in die Stadt hineinversetzt, vielleicht nicht wie in einer Zeitreise, aber doch mit einer verblüffenden Nähe. Die Neuinszenierung Yadegar Asisis macht auch deutlich, dass man dabei vergangenen Glanz empfindet. Schön - wahr - richtig: in seiner Zeit. Abgesehen von den Möglichkeiten der Digitalisierung ist Asisis Verfremdung durch die Montage von Fotos die einzig adäquate Form der Überarbeitung. Schon Gismondi hatte gemerkt, dass eine solche monumentale Schöpfung nicht mit archäologischen Korrekturen, verbessert' werden kann. Anders als von Reber meinte, war und ist der Spielraum bei der Rekonstruktion eines Stadtbildes keineswegs gering. Die wissenschaftlich gesehen,weißen Flecken' in der Romkarte sind viel größer, als man auf den ersten Blick glauben mag. Erst jetzt, aus einer Distanz von mehr als hundert Jahren, wird augenscheinlich, wie sehr Bühlmanns Rom eine Stadt der Beaux-Arts Architektenschule ist - und das sollte sie auch bleiben.

${ }^{74} \mathrm{Zu}$ diesem Modell: P. Ciancio Rossetto, La Reconstitution de Rome antique. Du plan-relief à celui de Gismondi, in: F. Hinard - M. Royo (Hrsg.), Rome. L'espace urbain \& ses représentations (Paris 1991) 237-256; G. Pisani Sartorio, Le plan-relief d'Italo Gismondi. Méthodes, techniques de réalisation et perspectives futurs, in: ebenda 257-277; C. F. Giuliani, Piani di lavoro per il Plastico di Roma, in: Filippi (Anm. 73) 261-265; siehe hier die Beiträge Cain, Bauer, Altekamp und Wulf-Rheidt mit Taf. 18b.

75 Die Fotos des Leporello wurden später nochmals benutzt von F. L. Dunbar, Rom. Sechshundert Bauwerke der Ewigen Stadt. Mit 26 Karten, 224 Abbildungen und 76 Zeichnungen nach Marten van Heemskerck (Berlin 1943). Wie weit dieses für ein großes Publikum gedachte Werk verbreitet war, ist mir nicht bekannt. 
Abbildungsnachweis

Abb. 1: Nach: Luigi Pirazzoli, Luigi Rossini 1790-1856 (Ravenna 1990) 190-191

Abb. 2: Niedersächsische Staats- und Universitätsbibliothek Göttingen, Sign. GR 2 H ITAL I,

324/31, Foto Stephan Eckardt

Abb. 3: Bibliothek der Soprintendenza Archeologica di Napoli, Foto Massimo Velo

Abb. 4: Universitätsbibliothek Augsburg, Foto Bibliothek

Abb. 5-6: Architekturmuseum der TU München, Foto Museum

Abb. 7: Foto Wulf-Rheidt

Abb. 8: Foto Soprintendenza Archeologica di Roma

Für die digitale Zusammenstellung der Einzelbilder sorgte D. Stante.

\section{Tafelnachweis}

Taf. 8a: Museen der Hansestadt Lübeck, Fotos Michael Kromat

Taf. 8b: Institut für Ägyptologie der Universität München, Foto Roy Hessing

Taf. 9: Architekturmuseum der TU München, Foto Museum 

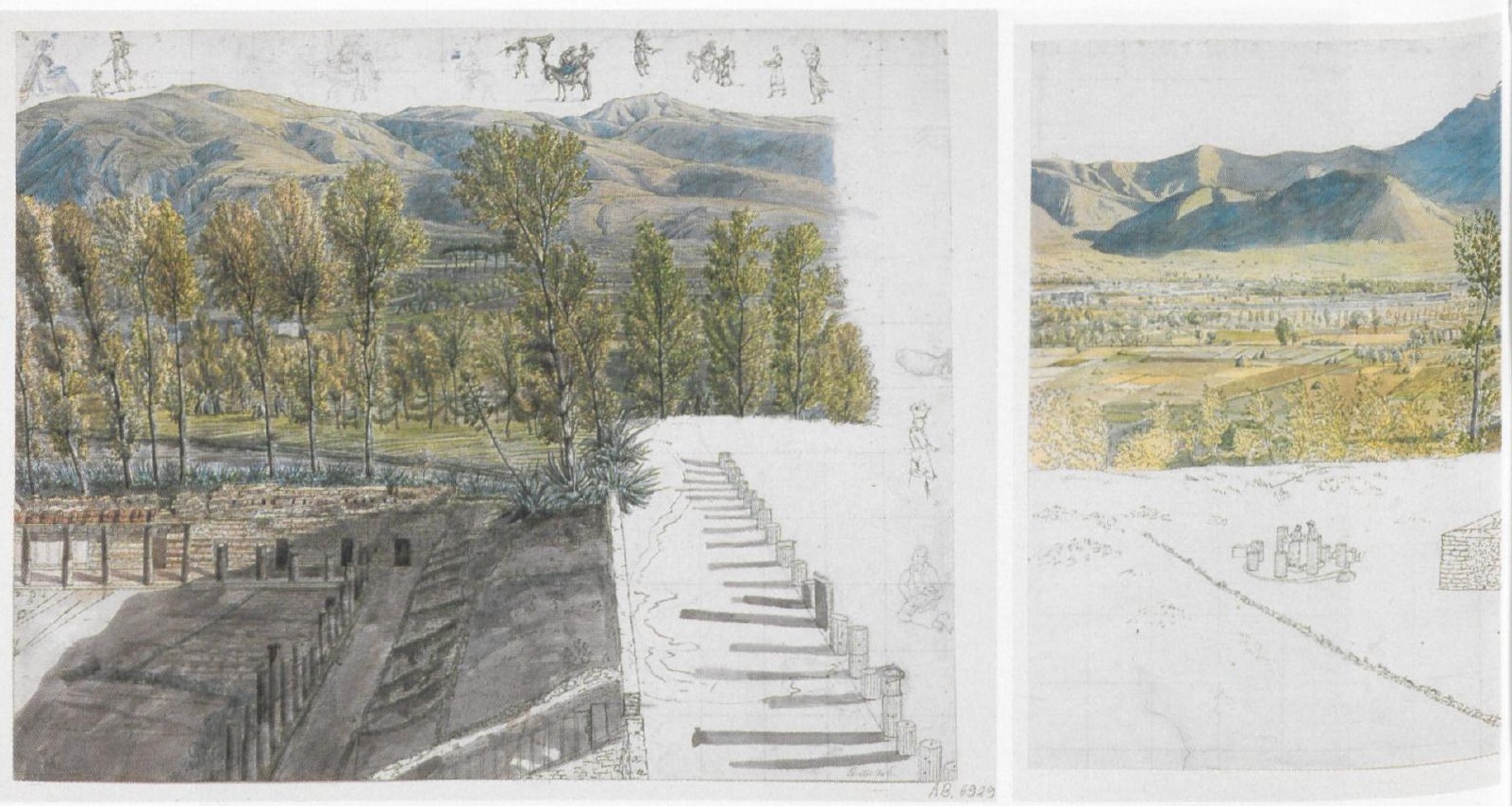

Taf. 8a Carl Georg Enslen, Drei Blätter aus dem Theaterpanorama von Pompeji, 1826

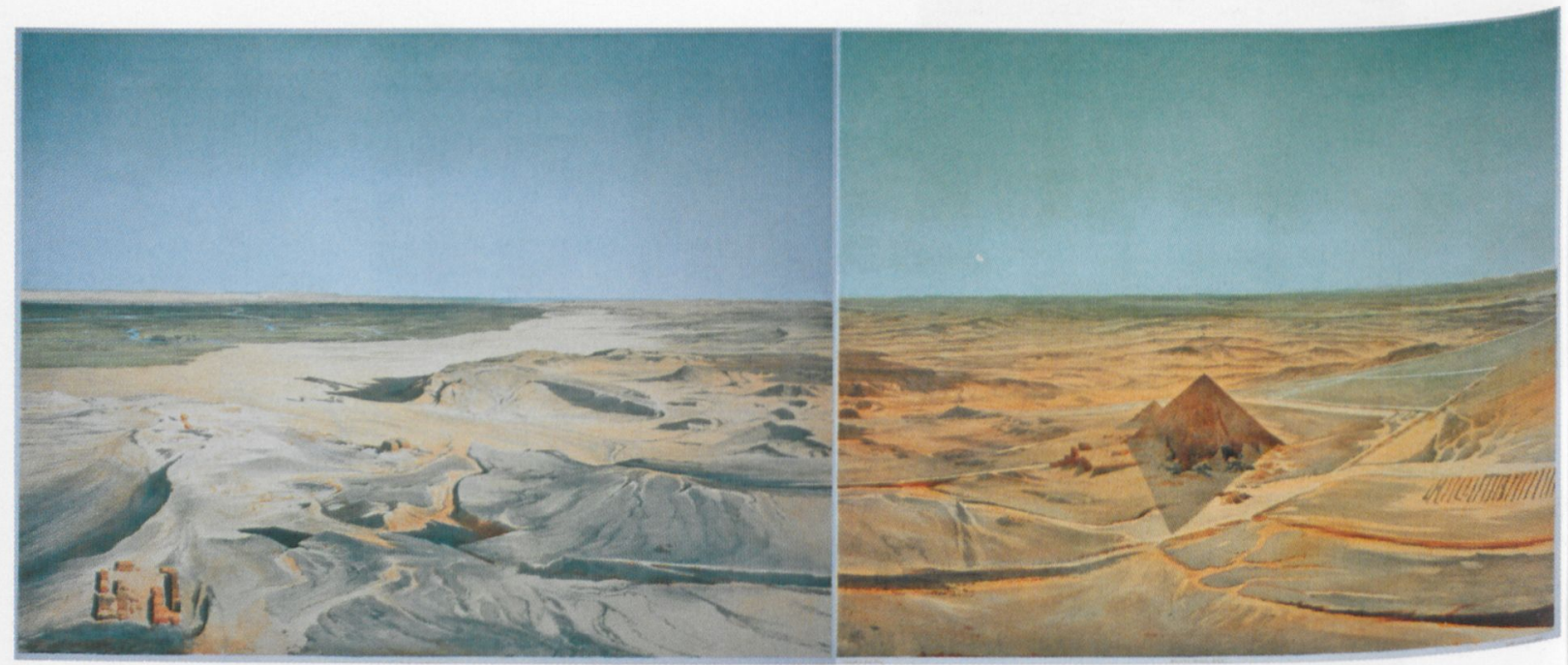

Taf. 8b Johann Jakob Frey oder Joseph Bonomi, Panorama von Gizeh, 1842, nach Richard Lepsius 


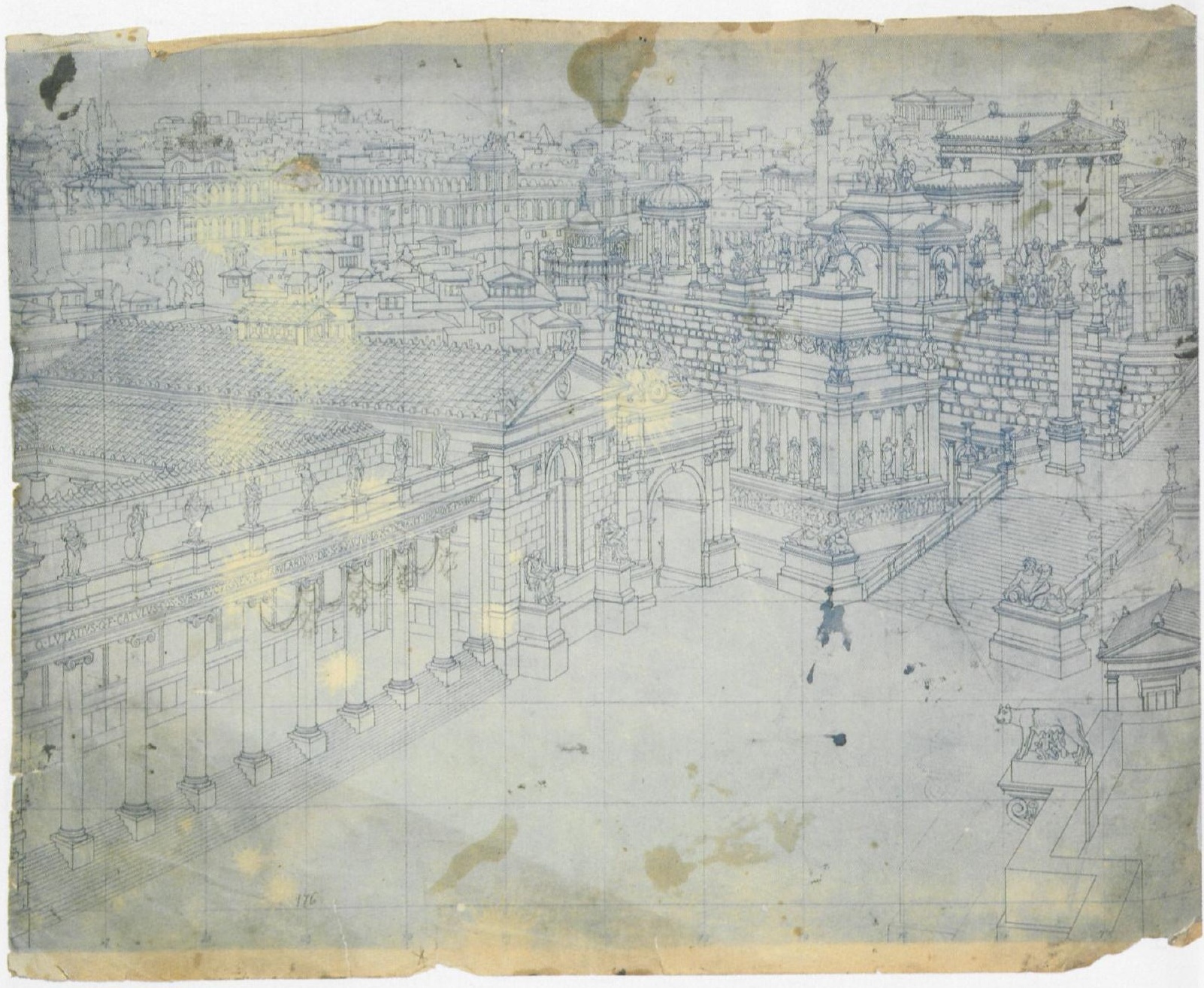

Taf. 9 Josef Bühlmann, Zwei Lichtpausen zur Vorbereitung des Rompanoramas mit Darstellung des Capitolhügels, um 1886. Architekturmuseum der TU München 


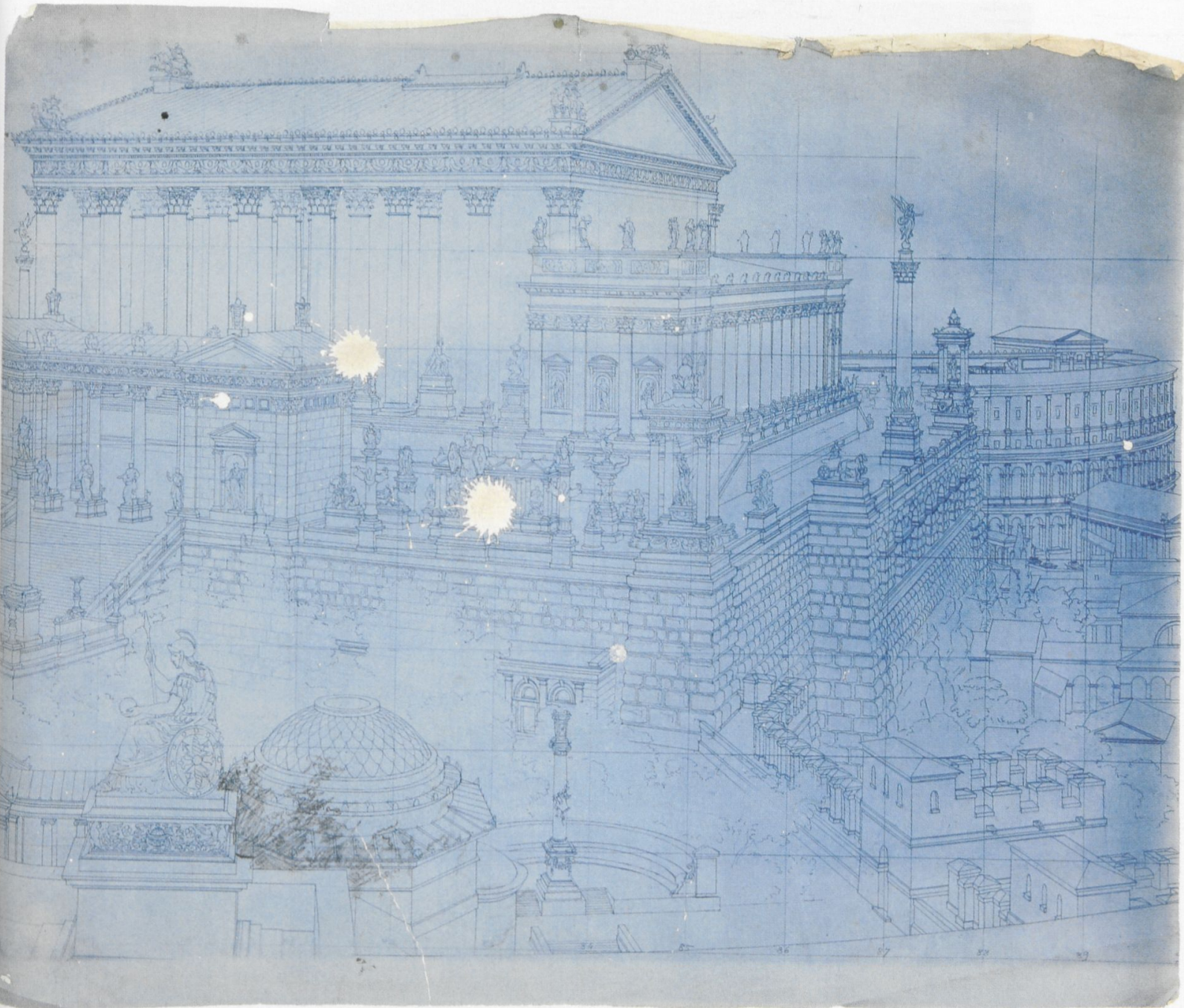




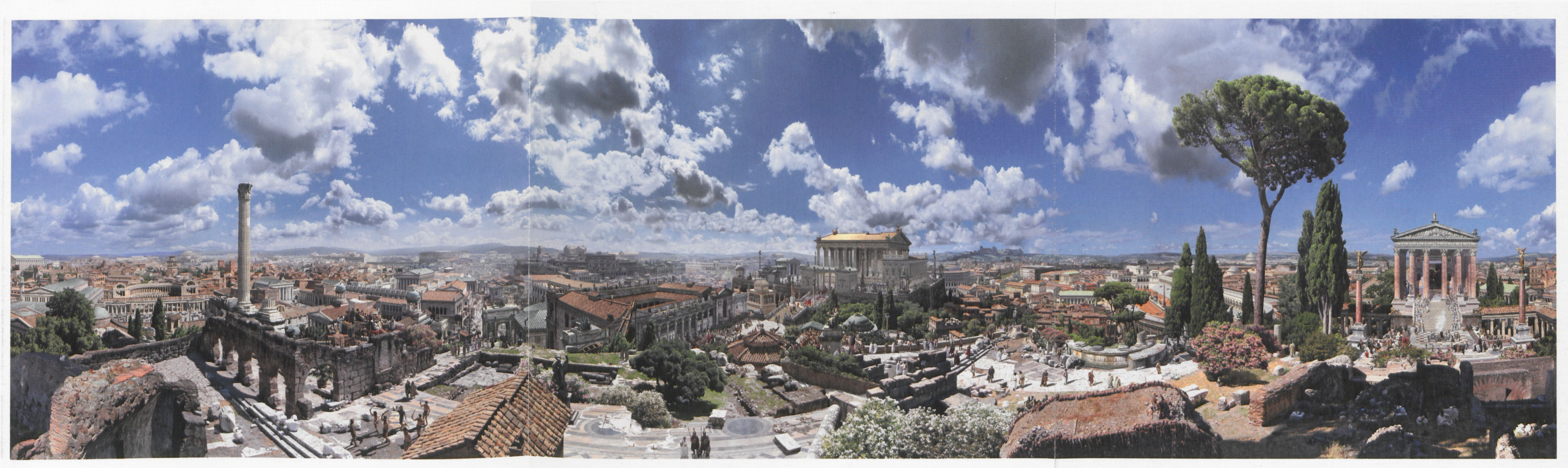

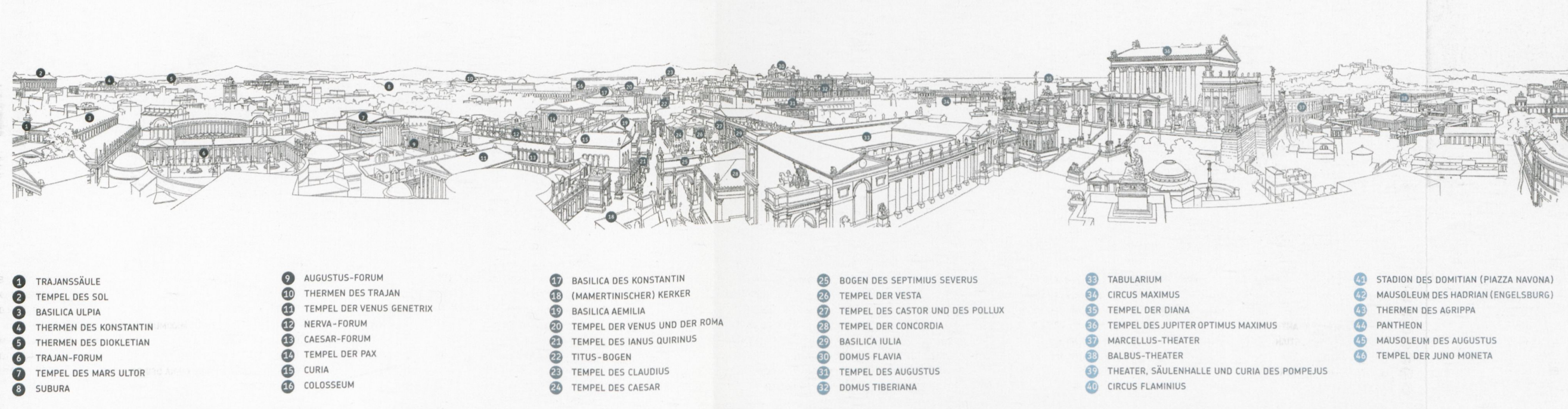

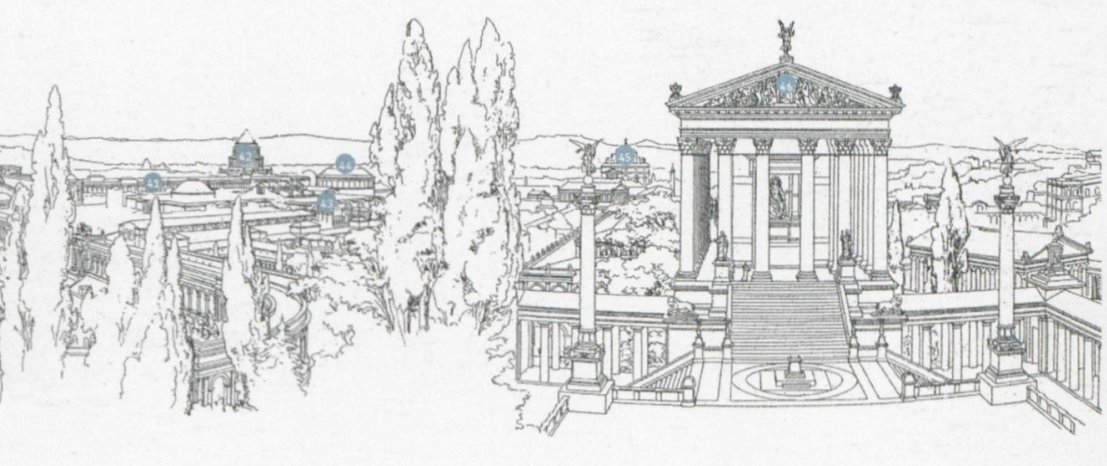

